\title{
Euler equations with several independent pressure laws and entropy satisfying explicit projection schemes
}

\author{
C. Chalons *and F. Coquel ${ }^{\dagger}$
}

October 24, 2005

\begin{abstract}
This work aims at numerically approximating the entropy weak solutions of Euler-like systems asymptotically recovered from the multi-pressure Navier-Stokes equations in the regime of an infinite Reynolds number. The non conservation form of the limit PDE model makes the shock solutions to be sensitive with respect to the underlying small scales. Here we propose to encode these small scale effects via a set of generalized jump conditions expressed in terms of the independent internal energies. The interest in considering internal energies stems from the presence of solely first order non conservative products by contrast to other variables. These non conservative products are defined in the now classical sense proposed by Dal Maso, LeFloch and Murat. We show how to enforce the generalized jump conditions at the discrete level with a fairly simple numerical procedure. This method is proved to satisfy a full set of stability estimates and to produce approximate solutions in good agreement with exact Riemann solutions.
\end{abstract}

\section{Introduction}

This work is devoted to the numerical approximation of the solutions of the Navier-Stokes equations with several independent pressure laws, in the regime of an infinite Reynolds number. Here, $N \geq 2$ pressure laws enter the PDE model via $N$ independent governing equations. The sum of the $N$ partial pressures determines the total pressure of the fluid. The systems under consideration are nothing but a natural extension of the classical Navier-Stokes equations (i.e. with $N=1$ ) and several models from the physics (plasma physics, turbulence) actually enter the present framework (see for instance[1],[4]). In many situations, these models come with a very large Reynolds number. Therefore and away from some boundaries, it sounds natural to neglect the small scales due to the viscous perturbations so as to consider some asymptotic Euler system in the limit of an infinite Reynolds number. However, in spite of the fact that these models exhibit close relashionships with the usual setting, a very difference arises when addressing the case $N \geq 2$. Namely the systems under consideration are then generally in non conservation form in the sense that non conservative products will always persist through any given admissible change of variables. Such non conservative products will involve either the unknown with its first order derivative or the unknown with its second order derivative, but generally speaking both types of products do coexist. The systems under study take the following condensed form :

$$
\partial_{t} \mathbf{V}^{\epsilon}+\mathbf{A}\left(\mathbf{V}^{\epsilon}\right) \partial_{x} \mathbf{V}^{\epsilon}=\epsilon \mathcal{R}\left(\mathbf{V}^{\epsilon}, \partial_{x}\left(\mathcal{B}\left(\mathbf{V}^{\epsilon}\right) \partial_{x} \mathbf{V}^{\epsilon}\right)\right)
$$

\footnotetext{
*Université Paris 7 and Laboratoire JLL, U.M.R. 7598, Boîte courrier 187, 75252 Paris Cedex 05, France E-mail: chalons@math.jussieu.fr

${ }^{\dagger}$ Centre National de la Recherche Scientifique and Laboratoire JLL, U.M.R. 7598, Boîte courrier 187, 75252 Paris Cedex 05, France E-mail: coquel@ann.jussieu.fr
} 
where the rescaling parameter $\epsilon>0$ stands as the inverse of the Reynolds number. The lack of full conservation form forbids us from using the classical distributions theory for defining the limit solutions as $\epsilon \rightarrow 0$. Several theories have been developed to handle the singular limit and we quote for instance the works by Dal Maso, LeFloch and Murat[11], LeFloch[18], Colombeau and Leroux[10], and more recently by Berthon, Coquel and LeFloch [3]. For our forthcoming numerical purposes, the problematic discussed hereafter turns out to fall in a very natural way within the mathematical framework proposed by LeFloch[18]. Indeed, this author suggests to define shock solutions for the limiting system as the limit of travelling wave solutions of (1) as the rescaling parameter $\epsilon$ goes to zero (see also Raviart-Sainsaulieu[20]). Such a natural approach allows the definition of the singular non conservative products in the limit $\epsilon \rightarrow 0$ in terms of a fixed family of paths which coincide with the graph of the travelling wave solutions. We shall adopt this approach in the present work. But let us emphasize that all the works we have reported highlight a deep discrepancy with respect to the purely conservative setting with genuine non linearity, namely the definition of shock solutions is sensitive with respect to the small scales present in the viscous perturbations. Sensitivity occurs in the sense that non proportional diffusive tensors give rise to distinct shock solutions. Put in other words, the lack of an admissible change of variables which recasts the system in full conservation form makes the definition of the endpoints of the travelling wave solutions sensitive with respect to the shape of the diffusive tensor. This sensitiveness in the definition of discontinuous solutions is also known in other settings. Let us quote hyperbolic systems in conservation form but with non linear fields that are not genuinely non linear, or mixed hyperbolic-elliptic systems (again in conservation form) describing phase transformations (see[17],[15],[8], ...).

Due to the sensitiveness we have just put forward, the numerical approximation of shock solutions associated with (1) in the asymptotic regime $\epsilon \rightarrow 0$ is a challenging issue. The very difficulty stays in the fact that the precise shape of the diffusive tensor must be carefully accounted for. In terms of numerical methods, this means that the numerical viscosity and the exact one must fit in order to capture numerical solutions in agreement with the expected ones. Recent works have been devoted to this matter. We refer the reader to Hou and LeFloch[16] for an analysis in the scalar case. In[1], Berthon and Coquel consider the numerical approximation of the solutions of the Navier-Stokes equations (i.e. with fixed $\epsilon>0$ ) when addressing two pressure laws $(N=2)$. They prove that classical approaches like splitting strategies between the convective and the diffusive parts for approximating the solutions of the system under study actually fail. These methods produce approximate solutions which exhibit large errors with the exact ones, and the main reason comes from a too large discrepancy between the numerical dissipation mechanism and the exact one. In a loose sense, the precise shape of the diffusive tensor in (1) is not properly taken into account. To overcome this difficulty, Berthon and Coquel introduce a correction step, referred as a "non linear projection" step, to enforce for validity at the discrete level some generalized jump conditions. These conditions express, in terms of entropies, the dependence of the shock solutions with respect to the exact diffusive tensor when keeping a precise souvenir of all the corresponding small scales effects. In practice, this correction step consists in solving a non linear and scalar algebraic problem on each computational cell. The resulting numerical scheme (prediction step + correction step), named "non linear projection scheme", obeys all the required positivity preserving and stability properties (entropy inequalities, maximum principles, ...), and we refer the reader to[1] for some numerical experiments illustrating the correct design of this strategy. In[5], Chalons and Coquel propose an extension of this scheme when dealing with $N>2$ independent pressure laws. In this context, $(N-1)$ distinct generalized jump conditions that reflect the sensitiveness of the solutions to the diffusive tensor (still in terms of entropies) are enforced for validity at the discrete level by the mean of a non linear correction step. Once more, the procedure succeeds in capturing 
numerical solutions in agreement with exact ones while satisfying the same stability properties as in the setting $N=2$. But again at the expense of the need for solving a succession of local non linear algebraic problems.

To bypass these nonlinearities, we propose in the present work to enforce for validity the generalized jump conditions when expressed in terms of the internal energies instead of the entropies. The benefit of dealing with internal energies is that the correction step becomes fully explicit due to the linear dependence of the total energy with respect to the partial internal energies. As a consequence, we no longer have to solve local non linear algebraic problems. In addition, this new approach shares with the one proposed in[5] (and also in[1]) the same positivity preserving and stability properties.

The present paper is organized as follows. The first section describes the PDE systems under study with a special emphasis put on the consequences of the lack of an equivalent conservation form. In particular, we highlight that shock solutions associated with these systems (in the asymptotic regime $\epsilon \rightarrow 0$ ) actually depend on the precise shape of the corresponding diffusive tensors, via the ratios of the viscosities $\mu_{i} /\left(\sum_{j=1}^{N} \mu_{j}\right)$ with $i=1, \ldots, N$. To this purpose, we put forward the so-called generalized jump conditions and we introduce an equivalent formulation (for smooth solutions) of (1) with second order operator in full conservation form. The interest in considering this equivalent form stems from the fact that it entirely keeps memory of the small scales effects via the ratios of the viscosity laws. The second section is devoted to the study of the asymptotic regime $\epsilon \rightarrow 0$, when exhibiting the Euler like system which governs the limiting solutions of (1). With this aim and after LeFloch[18], we define shock solutions as the limit of traveling wave solutions of (1) as the rescaling parameter $\epsilon$ goes to zero. Section 4 presents a suitable numerical method for approximating the weak solutions of the limiting systems on the basis of the Godunov method. Its design principle is focused on properly dealing with the exact diffusive tensor, while ensuring stability properties with the benefit of a low numerical effort. Section 5 generalizes this scheme in the Approximate Riemann Solvers framework proposed by Harten, Lax and Van Leer[14]. To conclude, section 6 gives numerical evidences of the correct design of the scheme. We underline that discrete solutions are systematically compared with exact Riemman solutions studied for existence and uniqueness by Chalons and Coquel[7].

\section{The Mathematical Setting}

In this paper, we are interested in the numerical approximation of the solutions of the following system in non conservation form :

$$
\left\{\begin{array}{l}
\partial_{t} \rho^{\epsilon}+\partial_{x} \rho u^{\epsilon}=0, \\
\partial_{t} \rho u^{\epsilon}+\partial_{x}\left(\rho u^{\epsilon 2}+\sum_{i=1}^{N} p_{i}^{\epsilon}\right)=\epsilon \partial_{x}\left(\sum_{i=1}^{N} \mu_{i} \partial_{x} u^{\epsilon}\right), \\
\partial_{t} \rho \varepsilon_{i}^{\epsilon}+\partial_{x} \rho \varepsilon_{i} u^{\epsilon}+p_{i}^{\epsilon} \partial_{x} u^{\epsilon}=\epsilon \mu_{i}\left(\partial_{x} u^{\epsilon}\right)^{2}, \quad i=1, \ldots, N,
\end{array}\right.
$$

in the regime of a vanishing rescaling parameter $\epsilon>0$. The PDE system readily stands as a natural extension of the usual Navier-Stokes equations when a single pressure is involved in the momentum equation. Here, $N$ independent pressure laws occur and are governed via $N$ internal energies $\rho \varepsilon_{i}$. Several models from the physics actually enter the present framework since their associated PDE system take the form (2) but for a prescribed number $N$ of pressure laws. We refer the reader to[4] for a detailed presentation. The required closure equations are as follows. The internal energies are assumed to obey the second principle of the thermodynamics, i.e. $\rho \varepsilon_{i}$ with $i \in\{1, \ldots, N\}$ is associated with an entropy $\rho s_{i}$ solution of

$$
-T_{i} d s_{i}=d \varepsilon_{i}+p_{i} d \tau, \quad \tau=1 / \rho,
$$


such that the mapping $\left(\tau, s_{i}\right) \rightarrow \varepsilon_{i}\left(\tau, s_{i}\right)$ is strictly convex. Then we have from (3),

$$
p_{i}\left(\tau, s_{i}\right)=-\partial_{\tau} \varepsilon_{i}\left(\tau, s_{i}\right), \quad T_{i}\left(\tau, s_{i}\right)=-\partial_{s_{i}} \varepsilon_{i}\left(\tau, s_{i}\right),
$$

where the temperature $T_{i}$ is classically assumed to stay positive. As a well known consequence, the well defined mapping $\left(\tau, \varepsilon_{i}\right) \rightarrow s_{i}\left(\tau, \varepsilon_{i}\right)$ is strictly convex and so is also, with some little abuse in the notation, the mapping $\left(\rho, \rho \varepsilon_{i}\right) \rightarrow\left\{\rho s_{i}\right\}\left(\rho, \rho \varepsilon_{i}\right):=\rho s_{i}\left(\frac{1}{\rho}, \frac{\rho \varepsilon_{i}}{\rho}\right)$. Each pressure law $p_{i}$ will be assumed in addition to obey the general Weyl's conditions for real gases (see[13] for the details). For simplicity, the viscosity coefficients $\mu_{i}$ will be assumed to be non negative real constants such that $\sum_{i=1}^{N} \mu_{i}>0$. In what follows, we shall tacitly assumed that $\mu_{N}>0$ and this is actually the case up to some relabelling in the $N$ entropies.

To shorten the notations, the system (2) is given the following condensed form :

$$
\partial_{t} \mathbf{V}^{\epsilon}+\mathbf{A}\left(\mathbf{V}^{\epsilon}\right) \partial_{x} \mathbf{V}^{\epsilon}=\epsilon \mathcal{R}\left(\mathbf{V}^{\epsilon}, \partial_{x}\left(\mathcal{B}\left(\mathbf{V}^{\epsilon}\right) \partial_{x} \mathbf{V}^{\epsilon}\right)\right)
$$

where the unknown $\mathbf{V}^{\epsilon}$ is associated with the following natural phase space :

$$
\Omega=\left\{\mathbf{V}=\left(\rho, \rho u,\left\{\rho \varepsilon_{i}\right\}_{i=1, \ldots, N}\right) \in \mathbb{R}^{N+2} / \rho>0, \rho u \in \mathbb{R},\left\{\rho \varepsilon_{i}>0\right\}_{i=1, \ldots, N}\right\} .
$$

The definition of the tensor $\mathcal{B}$ in (4) follows when writing

$$
\mu_{i}\left(\partial_{x} u\right)^{2}=\mu_{i}\left(\partial_{x}\left(u \partial_{x} u\right)-u \partial_{x x} u\right) .
$$

Let us underline that the non conservative products entering the definition of (4) involve not only the unknown $\mathbf{V}$ with its first derivative but also the unknown with its second derivative as expressed by the identity (5). In other words, both the first order operator and second order perturbation naturally occur in non conservation form. Let us first state some elementary properties of the non linear first order system. By virtue of the convexity of the $\varepsilon_{i}\left(\tau, s_{i}\right)$ mappings with $i \in\{1, \ldots, N\}$, we have the following statement which easy proof is left to the reader :

Lemma 1 The first order underlying system in (2) is hyperbolic over the phase space $\Omega$ and admits the following three distinct eigenvalues :

$$
u-c, \quad u, \quad u+c \quad \text { with } \quad c^{2}=\sum_{i=1}^{N} c_{i}^{2}, \quad c_{i}^{2}=\tau^{2} \partial_{\tau \tau}^{2} \varepsilon_{i}\left(\tau, s_{i}\right)>0,
$$

where $u$ has $N$ order of multiplicity. Moreover, under the Weyl's assumptions, the two extreme fields are genuinely non linear while the intermediate fields associated with the eigenvalue $u$ are linearly degenerate. In addition, the latter admit the velocity $u$ and the total pressure $\sum_{i=1}^{N} p_{i}$ as two independent Riemann invariants.

The system under consideration exhibits close relationships with the usual setting of the NavierStokes equations with a single pressure law, but naturally writes in non conservation form. This rises the question of the existence of additional conservation laws for smooth solutions of (2) and this is precisely the matter of the next statement :

Proposition 1 Smooth solutions of (2) satisfy the following conservation law :

$$
\partial_{t} \rho E^{\epsilon}+\partial_{x} \rho H u^{\epsilon}=\epsilon \partial_{x}\left(\left(\sum_{i=1}^{N} \mu_{i}\right) u^{\epsilon} \partial_{x} u^{\epsilon}\right)
$$


where the total energy $\rho E$ and the total enthalpy $\rho H$ respectively read:

$$
\rho E=\frac{(\rho u)^{2}}{2 \rho}+\sum_{i=1}^{N} \rho \varepsilon_{i}, \quad \rho H=\rho E+\sum_{i=1}^{N} p_{i} .
$$

These solutions also obey the following $N$ entropy balance equations :

$$
\partial_{t} \rho s_{i}^{\epsilon}+\partial_{x} \rho s_{i} u^{\epsilon}=-\frac{\mu_{i}}{T_{i}^{\epsilon}}\left(\partial_{x} u^{\epsilon}\right)^{2}, \quad i=1, \ldots, N .
$$

Note from (8) that classical non linear tranformations in the $s_{i}$ yield further additional balance equations for governing $\varphi\left(s_{1}, \ldots, s_{N}\right)$ where $\varphi: \mathbb{R}^{N} \rightarrow \mathbb{R}$ denotes any given arbitrary smooth function. Nevertheless and without specific assumptions on the thermodynamic closure equations, none of these additional equations boils down to a non trivial additional conservation law. With this respect, the system (2) is genuinely in non conservation form. In order to put forward a deep consequence of the lack of conservation form, it is useful to illustrate the nature of the non trivial conservation laws coming with restrictive modelling assumptions. In that aim, we briefly discuss in the next subsection a particular setting for which the system (2) recasts in full conservation form but with the striking property that the limit system obtained in the asymptotic regime $\epsilon \rightarrow 0$ does keep an explicit souvenir of the vanishing viscous perturbation. Namely, the available additional conservation laws explicitly involve the ratios of the viscosity coefficients. Such a souvenir exactly expresses the property that the $N$ independent entropies cannot achieve independent jumps for shock solutions but on the contrary jumps that obey proportionality relations, the latter being precisely dictated by the ratios of the viscosities. In the general setting for (2), such proportionality conditions will obviously persist but in a weaker form and will be referred as to generalized jump conditions. We propose to point out this deep property on a simple example for its primary importance in the numerical approximation of the solutions of (2).

\section{$2.1 \quad$ A simplified setting}

Let us temporarily choose $N=2$ when considering a polytropic closure relation for defining each of the two independent pressure laws. Let us assume in addition the same constant adiabatic exponent, say $\gamma>1$, for the two pressures so that :

$$
p_{i}=(\gamma-1) \rho \varepsilon_{i}, \quad i=1,2 .
$$

Let us then complete the above equations of state when considering the following specific entropies

$$
s_{i}=p_{i} / \rho^{\gamma}, \quad i=1,2 .
$$

The interest of such a simplified setting finds its root in the following statement :

Proposition 2 Assuming the modelling assumptions (9)-(10), the smooth solutions of (2) obey equivalently the following system in full conservation form :

$$
\left\{\begin{array}{l}
\partial_{t} \rho^{\epsilon}+\partial_{x} \rho u^{\epsilon}=0 \\
\partial_{t} \rho u^{\epsilon}+\partial_{x}\left(\rho u^{\epsilon}+\sum_{i=1}^{2} p_{i}^{\epsilon}\right)=\epsilon \partial_{x}\left(\sum_{i=1}^{2} \mu_{i} \partial_{x} u^{\epsilon}\right), \\
\partial_{t} \rho E^{\epsilon}+\partial_{x}\left(\rho E^{\epsilon}+\sum_{i=1}^{2} p_{i}^{\epsilon}\right) u^{\epsilon}=\epsilon \partial_{x}\left(\sum_{i=1}^{2} \mu_{i} u^{\epsilon} \partial_{x} u^{\epsilon}\right), \\
\partial_{t} \rho\left(s_{1}-\frac{\mu_{1}}{\mu_{2}} s_{2}\right)^{\epsilon}+\partial_{x} \rho\left(s_{1}-\frac{\mu_{1}}{\mu_{2}} s_{2}\right) u^{\epsilon}=0 .
\end{array}\right.
$$




\section{Proof}

This result is a direct consequence of proposition 1 when noticing that (8) gives in the present simplified setting :

$$
\frac{1}{\mu_{i}}\left(\partial_{t} \rho s_{i}^{\epsilon}+\partial_{x} \rho s_{i} u^{\epsilon}\right)=\epsilon \frac{\gamma-1}{\rho^{\epsilon \gamma-1}}\left(\partial_{x} u^{\epsilon}\right)^{2}, \quad i=1,2 .
$$

Then substrating these two equations readily yields the last non trivial conservation law in (11). To conclude it suffices to notice that the mapping $\mathbf{V} \in \Omega \rightarrow^{T}\left(\rho, \rho u, \rho E, \rho\left(s_{1}-\frac{\mu_{2}}{\mu_{1}} s_{2}\right)\right)$ yields an admissible change of variables. As an immediate consequence of proposition 2 , we next state :

Corollary 2 Assuming that smooth solutions $\left\{\mathbf{V}^{\epsilon}\right\}_{\epsilon>0}$ of (11) converge strongly (in relevant topologies) as $\epsilon$ goes to 0 to a limit function $\mathbf{V}$, then $\mathbf{V}$ is a solution in sense of the distributions of the following non linear hyperbolic system :

$$
\left\{\begin{array}{l}
\partial_{t} \rho+\partial_{x} \rho u=0 \\
\partial_{t} \rho u+\partial_{x}\left(\rho u^{2}+\sum_{i=1}^{2} p_{i}\right)=0 \\
\partial_{t} \rho E+\partial_{x}\left(\rho E+\sum_{i=1}^{2} p_{i}\right) u=0 \\
\partial_{t} \rho\left(s_{1}-\frac{\mu_{1}}{\mu_{2}} s_{2}\right)+\partial_{x} \rho\left(s_{1}-\frac{\mu_{1}}{\mu_{2}} s_{2}\right) u=0
\end{array}\right.
$$

supplemented with the Lax entropy inequalities :

$$
\partial_{t} \rho h\left(s_{i}\right)+\partial_{x} \rho h\left(s_{i}\right) u \leq 0, \quad h\left(s_{i}\right)=-\ln \left(s_{i}\right), \quad i=1,2 .
$$

In the zone of smoothness of the limit solution, $\mathbf{V}$ satisfies in addition to the conservation laws for $\rho, \rho u$ and $\rho E$ :

$$
\partial_{t} \rho s_{i}+\partial_{x} \rho s_{i} u=0, \quad i=1,2,
$$

while and by contrast across shocks (i.e. discontinuities associated with the two extreme fields, see lemma 1), the limit solution must obey with classical notations the following Rankine-Hugoniot conditions :

$$
\left\{\begin{array}{l}
m=\rho^{-}\left(u^{-}-\sigma\right)=\rho^{+}\left(u^{+}-\sigma\right) \\
m\left(u^{+}-u^{-}\right)+\left(\sum_{i=1}^{2} p_{i}^{+}-\sum_{i=1}^{2} p_{i}^{-}\right)=0 \\
m\left(E^{+}-E^{-}\right)+\left(\left(\sum_{i=1}^{2} p_{i} u\right)^{+}-\left(\sum_{i=1}^{2} p_{i} u\right)^{-}\right)=0 \\
\left(s_{1}^{+}-s_{1}^{-}\right)=\frac{\mu_{1}}{\mu_{2}}\left(s_{2}^{+}-s_{2}^{-}\right)
\end{array}\right.
$$

supplemented with the entropy condition :

$$
m\left(h\left(s_{i}^{+}\right)-h\left(s_{i}^{-}\right)\right)<0, \quad i=1,2 .
$$

The smooth mapping $h$ entering the inequalities (14) and (17) is considered for the sake of convexity of the mapping $\left(\rho, \rho \varepsilon_{i}\right) \rightarrow\left\{\rho h\left(s_{i}\right)\right\}\left(\rho, \rho \varepsilon_{i}\right)$. Proof

Assuming a zone of smoothness for $\mathbf{V}$, classical manipulations on the momentum and total energy equations yield (see[13] in the setting of the usual Euler equations) :

$$
\partial_{t} \rho\left(\varepsilon_{1}+\varepsilon_{2}\right)+\partial_{x} \rho\left(\varepsilon_{1}+\varepsilon_{2}\right) u+\sum_{i=1}^{2} p_{i} \partial_{x} u=0,
$$

that is to say, in view of $(3)$ :

$$
\partial_{t} \rho\left(s_{1}+s_{2}\right)+\partial_{x} \rho\left(s_{1}+s_{2}\right) u=0,
$$


and henceforth the expected identities (15) when invoking the last conservation law in (13). Turning now considering shock solutions, we just underline that the Rankine-Hugoniot conditions coming with (13) clearly give :

$$
-\sigma\left(\rho\left(s_{1}-\frac{\mu_{1}}{\mu_{2}} s_{2}\right)\right)+\left(\rho\left(s_{1}-\frac{\mu_{1}}{\mu_{2}} s_{2}\right) u\right)=0
$$

which is easily seen to boil down to the expected one in (16) by virtue of a non zero relative mass flux $m$ for the discontinuities under consideration (again see[13] for the details). Let us briefly rephraze the above results. First the conservation of the entropies (15) in the zone of smoothness of the limit solution is a well-known and expected property. The breakdown of conservation (17) for shock solutions is also well-known but the striking novelty here stays in the property that the jumps in the two specific entropies must be kept in balance according to the ratio of the two viscosities. This unusual Rankine-Hugoniot condition obviously implies that the shock solutions propagating with speed $\sigma$ and separating two constant states $\mathbf{V}^{-}$and $\mathbf{V}^{+}$have the property that $\mathbf{V}^{+}$does not only depend on $\sigma$ and $\mathbf{V}^{-}$but also on the ratio of the viscosities $\mu_{1} / \mu_{2}$ : i.e $\mathbf{V}^{+}:=$ $\left\{\mathbf{V}^{+}\right\}\left(\sigma, \mathbf{V}^{-}, \mu_{1} / \mu_{2}\right)$. In other words, $\sigma$ and $\mathbf{V}^{-}$being fixed, modifying the ratio $\mu_{1} / \mu_{2}$ does modify $\mathbf{V}^{+}$. Such a dependency is actually natural and illustrates a general property met by shock solutions for hyperbolic systems in non conservation form (see[18],[11],[20] and below).

\section{$2.2 \quad$ The general setting}

Let us turn back to the general setting for which (2) is genuinely in non conservation form. Actually and as shown below, this system can be given two convenient equivalent forms for smooth solutions with the following distinctive properties. A first formulation which allows to recast the first order operator in conservation form but not its second order counterpart. Then a second formulation where by contrast the second order operator achieves a conservation form to the detriment of the first order one. Both formulations are deeply motivated by the need to track closely the ratios of the viscosity coefficients in the governing equations. More precisely, both formulations intend to model the property that the rates of dissipation governing each of the entropies $\rho s_{i}, i \in\{1, \ldots, N\}$, in their evolution in time, are actually kept in balance according to such ratios.

The first formulation readily follows from proposition 1 when considering the admissible change of variables given by :

$$
\mathbf{U}:=\mathbf{U}(\mathbf{V})=\left(\rho, \rho u,\left\{\rho s_{i}\right\}_{i=1, \ldots, N}\right),
$$

so that $(2)$ is given the following equivalent form :

$$
\partial_{t} \mathbf{U}^{\epsilon}+\partial_{x} \mathbf{F}\left(\mathbf{U}^{\epsilon}\right)=\epsilon \mathcal{R}\left(\mathbf{U}^{\epsilon}, \partial_{x}\left(\mathcal{C}\left(\mathbf{U}^{\epsilon}\right) \partial_{x} \mathbf{U}^{\epsilon}\right)\right) .
$$

Here and with clear notations, the flux function $\mathbf{F}: \Omega \rightarrow \mathbb{R}^{N+2} \operatorname{reads} \mathbf{F}(\mathbf{U})=\left(\rho u, \rho u^{2}+\right.$ $\left.\sum_{i=1}^{N} p_{i},\left\{\rho s_{i} u\right\}_{i=1, \ldots, N}\right)$ while the diffusive tensor $\mathcal{C}\left(\mathbf{U}^{\epsilon}\right)$ finds a clear definition (see indeed (5)). Let us then underline that the $N$ entropy balance equations (8) entering the definition of (19) do not evolve independently but actually proportionally according to :

$$
\frac{\mu_{N}}{T_{N}^{\epsilon}}\left(\partial_{t} \rho s_{i}^{\epsilon}+\partial_{x} \rho s_{i} u^{\epsilon}\right)=\frac{\mu_{i}}{T_{i}^{\epsilon}}\left(\partial_{t} \rho s_{N}^{\epsilon}+\partial_{x} \rho s_{N} u^{\epsilon}\right), \quad 1 \leq i \leq N-1 .
$$

These identities simply reflect a (trivial) cancellation property in $\left(\partial_{x} u^{\epsilon}\right)^{2}$ we have already used within the simplified setting. Of course, (20) is nothing but a natural extension of the non trivial conservation law built on the ratio of the viscosities in the system (11). Next, let us observe that the smooth solutions of (2) obviously satisfy the additional conservation law (7) for governing the total energy $\rho E$ :

$$
\partial_{t}\{\rho E\}\left(\mathbf{U}^{\epsilon}\right)+\partial_{x}\{\rho H u\}\left(\mathbf{U}^{\epsilon}\right)=\epsilon \partial_{x}\left(\sum_{i=1}^{N} \mu_{i} u^{\epsilon} \partial_{x} u^{\epsilon}\right)
$$


The equalities (20) and (21) stay at the basis of the work[5] (see also[4]) where a natural generalization of the system (11) to the general setting is proposed. Such an extension has been proved to be successful in the numerical approximation of the solutions of (19) in the limit $\epsilon \rightarrow 0$ which we again emphasize to be heavily driven by the property that entropy rates of dissipation must be kept in balance according to (20). At the cornerstone of this success is a non linear correction procedure for (19) which aims at enforcing for validity at the discrete level the $(N-1)$ identities in (20) while ensuring the conservation of the total energy in (21). Roughly speaking, the non linearities in the procedure just reflect the non linearities in the total energy $\rho E$ when expressed in terms of the entropies $\left\{\rho s_{i}\right\}_{1 \leq i \leq N}$ (see[5],[4] for the details). Observing by contrast that the total energy $\rho E$ does linearly depend on the internal energies $\left\{\rho \varepsilon_{i}\right\}_{1 \leq i \leq N}$ strongly promotes to introduce an alternative formulation to (19), now built on the internal energies but which mimics the key equalities (20). The second principle of the thermodynamics (3) obviously allows for this, since it equivalently recasts $(20)$ as :

$$
\begin{gathered}
\mu_{N}\left(\partial_{t} \rho \varepsilon_{i}^{\epsilon}+\partial_{x} \rho \varepsilon_{i} u^{\epsilon}+p_{i}^{\epsilon} \partial_{x} u^{\epsilon}\right)=\mu_{i}\left(\partial_{t} \rho \varepsilon_{N}^{\epsilon}+\partial_{x} \rho \varepsilon_{N} u^{\epsilon}+p_{N}^{\epsilon} \partial_{x} u^{\epsilon}\right) \\
1 \leq i \leq N-1
\end{gathered}
$$

Therefore and since the viscosities are constant real numbers, we get :

$$
\begin{gathered}
\partial_{t} \rho\left(\varepsilon_{i}-\frac{\mu_{i}}{\mu_{N}} \varepsilon_{N}\right)^{\epsilon}+\partial_{x} \rho\left(\varepsilon_{i}-\frac{\mu_{i}}{\mu_{N}} \varepsilon_{N}\right) u^{\epsilon}+\left(p_{i}-\frac{\mu_{i}}{\mu_{N}} p_{N}\right)^{\epsilon} \partial_{x} u^{\epsilon}=0, \\
1 \leq i \leq N-1 .
\end{gathered}
$$

These identities then suggest us to introduce :

Lemma 3 The following mapping :

$$
\begin{gathered}
\mathbf{W}:=\mathbf{W}(\mathbf{V})=\left(\rho, \rho u, \rho E,\left\{\rho w_{i}\right\}_{i=1, \ldots, N-1}\right), \\
\rho w_{i}=\rho\left(\varepsilon_{i}-\frac{\mu_{i}}{\mu_{N}} \varepsilon_{N}\right), \quad 1 \leq i \leq N-1,
\end{gathered}
$$

yields an admissible change of variables which equivalently recasts the system (2) under the following form :

$$
\partial_{t} \mathbf{W}^{\epsilon}+\mathbf{B}\left(\mathbf{W}^{\epsilon}\right) \partial_{x} \mathbf{W}^{\epsilon}=\epsilon \partial_{x}\left(\mathcal{D}\left(\mathbf{W}^{\epsilon}\right) \partial_{x} \mathbf{W}^{\epsilon}\right),
$$

where $\mathbf{B}$ and $\mathcal{D}$ find clear definitions from (23) and (7).

This result simply follows when noticing that :

$$
\left\{\rho \varepsilon_{i}\right\}(\mathbf{W})=\rho w_{i}+\frac{\mu_{i}}{\sum_{j=1}^{N} \mu_{j}}\left(\rho E-\frac{(\rho u)^{2}}{2 \rho}-\sum_{j=1}^{N-1} \rho w_{j}\right), \quad 1 \leq i \leq N-1,
$$

while of course

$$
\left\{\rho \varepsilon_{N}\right\}(\mathbf{W})=\rho E-\frac{(\rho u)^{2}}{2 \rho}-\sum_{j=1}^{N-1} \rho \varepsilon_{j}(\mathbf{W}) .
$$

Let us again emphasize that the interest in considering the equivalent form (25) over the original one (2) stems from the fact that the balance to be kept in the entropy rates of dissipation is built in but when expressed in terms of the internal energies : namely (22). With this respect, the form (25) will be shown to be particularly suited for our numerical purposes. Since we are interested in the asymptotic regime of a vanishing parameter $\epsilon$, we propose to make meaningfull in the next section the identities (22) and thus the change of variables (24) in the limit $\epsilon \rightarrow 0$. 


\section{The Asymptotic Regime}

This section aims at exhibiting the asymptotic first order system for governing limit solutions of (2) in the regime $\epsilon \rightarrow 0$. This system will arise as a natural extension of the limit model (13) obtained under simplifying assumptions. We also extend here the equivalence lemma 3 between the two formulations (2) and (25) to a class of discontinuous functions which naturally arise in the limit $\epsilon \rightarrow 0$. Here, we specifically focus ourselves on piecewise Lipschitz continuous functions.

Let us first motivate the forthcoming developments when formally addressing the asymptotic regime $\epsilon \rightarrow 0$ in the non linear system (2). As it is well known, solutions of the underlying hyperbolic system may develop discontinuities in finite time from smooth initial data. As a consequence, families of smooth solutions $\left\{\mathbf{V}^{\epsilon}\right\}_{\epsilon>0}$ fail generally speaking to converge to smooth functions and this first rises the question of the singular limit in the non conservative products. Condidering at first $\mathbf{A}\left(\mathbf{V}^{\epsilon}\right) \partial_{x} \mathbf{V}^{\epsilon}$ and assuming a piecewise Lipschitz continuous limit function, such a product coincides with a bounded Borel measure. Similarly and obviously, the $\epsilon$-weighted non conservative product $\epsilon\left(\partial_{x} u^{\epsilon}\right)^{2}$ does not vanish with $\epsilon$ in general but rather converges to a non negative bounded measure, say $\mathcal{N}$. We are thus led to consider the following system for governing the limit solution V:

$$
\left\{\begin{array}{l}
\partial_{t} \rho+\partial_{x} \rho u=0, \\
\partial_{t} \rho u+\partial_{x}\left(\rho u^{2}+\sum_{i=1}^{N} p_{i}\right)=0, \\
\partial_{t} \rho \varepsilon_{i}+\partial_{x} \rho \varepsilon_{i} u+\left[p_{i} \partial_{x} u\right]_{\phi}=\mu_{i} \mathcal{N}, \quad i=1, \ldots, N .
\end{array}\right.
$$

Obviously, the Borel measure $\mathcal{N}$ in (26) is identically zero in the smooth regions of the limit function $\mathbf{V}$ and is expected to exhibit a non trivial mass along the discontinuities of $\mathbf{V}$. Two distinct types of discontinuities are in order. First, contact discontinuities coming with the intermediate LD fields propagate with constant speed $u$. Hence, both the measures $\left[p_{i} \partial_{x} u\right]_{\phi}$ and $\mathcal{N}$ identically vanish in such discontinuities. By contrast, these measures have a non trivial mass along the shock discontinuities associated with the two extreme GNL fields since the velocity $u$ achieves a non trivial jump. In order to define these measures, we propose following LeFloch[18] to define shock solutions in the extreme fields as the limit as $\epsilon$ goes to zero of Travelling Wave (TW) solutions of (2). Let us briefly recall that a travelling wave is a smooth solution of (2) (and thus of the equivalent form (25)) of the special form $\mathbf{V}_{\epsilon}(x, t)=\mathcal{V}_{\epsilon}(x-\sigma t)=\mathcal{V}_{\epsilon}(\xi)$ which satisfies $\lim _{\xi \rightarrow \pm \infty} \mathcal{V}_{\epsilon}(\xi)=\mathbf{V}^{ \pm}$with $\lim _{\xi \rightarrow \pm \infty} d_{\xi} \mathcal{V}_{\epsilon}(\xi)=0$. Next, introducing the function $\mathcal{V}: \mathbb{R} \rightarrow \mathbb{R}$ defined by $\mathcal{V}\left(\frac{\xi}{\epsilon}\right)=\mathcal{V}_{\epsilon}(\xi)$, then $\mathcal{V}$ is solution of the following ODE problem, free from the rescaling parameter $\epsilon$ :

$$
\left\{\begin{array}{l}
-\sigma d_{\xi} \rho+d_{\xi} \rho u=0, \\
-\sigma d_{\xi} \rho u+d_{\xi}\left(\rho u^{2}+\sum_{i=1}^{N} p_{i}\right)=d_{\xi}\left(\sum_{i=1}^{N} \mu_{i} d_{\xi} u\right), \\
-\sigma d_{\xi} \rho \varepsilon_{i}+d_{\xi} \rho \varepsilon_{i} u+p_{i} d_{\xi} u=\mu_{i}\left(d_{\xi} u\right)^{2}, i=1, \ldots, N,
\end{array}\right.
$$

with $\lim _{\xi \rightarrow \pm \infty} \mathcal{V}(\xi)=\mathbf{V}^{ \pm}, \lim _{\xi \rightarrow \pm \infty} d_{\xi} \mathcal{V}(\xi)=0$. For any given state $\mathbf{V}^{-} \in \Omega$ and velocity $\sigma$ properly prescribed under the classical condition $\sigma<u_{-}-c\left(\mathbf{V}^{-}\right)$, existence and uniqueness (up to some translation) of a TW solution for the first field is proved in[3],[7] (see also[2]) (the case of the other extreme field immediately follows from Galilean invariance). Due to the non conservation form met by these equations, let us emphasize that the exit state $\mathbf{V}^{+}$not only depends on $\sigma$ and $\mathbf{V}^{-}$but also on the ratios of the viscosities $\left\{\mu_{i} / \sum_{i=1}^{N} \mu_{i}\right\}_{i=1, \ldots, N}$. Chalons and Coquel in[7] show how to recover the state $\mathbf{V}^{+}$in the setting of $N$ polytropic gas laws when solving a scalar nonlinear equation built on the above viscosity ratios.

Equipped with the family of smooth solutions $\left\{\mathbf{V}_{\epsilon}\right\}_{\epsilon>0}$, we classically observe that for all $\epsilon>0$ :

$$
\left\|d_{\xi} \mathcal{V}_{\epsilon}\right\|_{L^{1}(\mathbb{R})}=\left\|d_{\xi} \mathcal{V}\right\|_{L^{1}(\mathbb{R})}<\infty
$$


so that the family $\left(\mathcal{V}_{\epsilon}\right)_{\epsilon>0}$ converges in the $L_{l o c}^{1}$ topology in the limit $\epsilon \rightarrow 0$ to the following discontinuous function :

$$
\mathbf{V}(x, t)= \begin{cases}\mathbf{V}^{-} & \text {if } x<\sigma t \\ \mathbf{V}^{+} & \text {if } x>\sigma t\end{cases}
$$

which by definition[18] is said to be a shock solution of (33) (see also[20]). We are now in position to exhibit the jump conditions satisfied by the shock solution (28) under consideration. In that aim, let us deduce from (27) after integration along the profile :

$$
\begin{aligned}
& m:=\rho^{-}\left(u^{-}-\sigma\right)=\rho^{+}\left(u^{+}-\sigma\right), \\
& m\left(u^{+}-u^{-}\right)+\left(\sum_{i=1}^{N} p_{i}^{+}-\sum_{i=1}^{N} p_{i}^{-}\right)=0, \\
& m\left(\varepsilon_{i}^{+}-\varepsilon_{i}^{-}\right)+\int_{\mathbb{R}} p_{i} d_{\xi} u d \xi=\mu_{i} \int_{\mathbb{R}}\left(d_{\xi} u\right)^{2} d \xi .
\end{aligned}
$$

These identities now allow us to define the Borel measures $\left[p_{i} \partial_{x} u\right]_{\phi}$ and $\mathcal{N}$ in (26). To that purpose, it is convenient to define after LeFloch[18] the family of paths $\phi$ when setting

$$
\phi\left(s ; \mathbf{V}^{-}, \mathbf{V}^{+}\right)=\mathcal{V}(\Phi(s)),
$$

where $\Phi$ is an increasing smooth bijection from $] 0,1[$ onto $\mathbb{R}$. Let us underline that (30) is invariant under reparametrization. Then across a shock discontinuity propagating with speed $\sigma$ and separating two states $\mathbf{V}^{-}$and $\mathbf{V}^{+}$, the mass of the Borel measures under consideration respectively reads :

$$
\left[p_{i} \partial_{x} u\right]_{\phi}=\int_{0}^{1} p_{i}\left(\phi\left(s ; \mathbf{V}^{-}, \mathbf{V}^{+}\right)\right) \partial_{s} u\left(\phi\left(s ; \mathbf{V}^{-}, \mathbf{V}^{+}\right)\right) d s \delta_{x-\sigma t}, \quad 1 \leq i \leq N
$$

and

$$
\mathcal{N}=\int_{0}^{1}\left|\partial_{s} u\left(\phi\left(s ; \mathbf{V}^{-}, \mathbf{V}^{+}\right)\right)\right|^{2} d s \delta_{x-\sigma t}
$$

With clear notations, we shall use in the sequel the following condensed form :

$$
\partial_{t} \mathbf{V}+\left[\mathbf{A}(\mathbf{V}) \partial_{x} \mathbf{V}\right]_{\phi}=\mathbf{N}(\mathbf{V})
$$

with

$$
\mathbf{N}(\mathbf{V})=\left(\left\{N_{i}(\mathbf{V})\right\}_{i=1, \ldots, N}\right), \quad N_{i}(\mathbf{V})=\mu_{i} \mathcal{N}(\mathbf{V}), \quad i=1, \ldots, N .
$$

Next turning considering the formulation (25), the limit system is obtained along the same lines when noticing that both (25) and (2) admit the same travelling waves in view of the smoothness of these solutions. In other words, both family of shock solutions are identical. In the framework of piecewise Lipschitz continuous solutions, the limit system can be thus given the following form :

$$
\left\{\begin{array}{l}
\partial_{t} \rho+\partial_{x} \rho u=0 \\
\partial_{t} \rho u+\partial_{x}\left(\rho u^{2}+\sum_{i=1}^{N} p_{i}\right)=0 \\
\partial_{t} \rho E+\partial_{x}\left(\rho E+\sum_{i=1}^{N} p_{i}\right) u=0, \\
\partial_{t} \rho w_{i}+\partial_{x} \rho w_{i} u+\left[\left(p_{i}-\frac{\mu_{i}}{\mu_{N}} p_{N}\right) \partial_{x} u\right]_{\phi}=0, \quad i=1, \ldots, N-1,
\end{array}\right.
$$

or again the following condensed form :

$$
\partial_{t} \mathbf{W}+\left[\mathbf{B}(\mathbf{W}) \partial_{x} \mathbf{W}\right]_{\phi}=0,
$$

supplemented by the Lax entropy inequalities :

$$
\partial_{t} \rho s_{i}+\partial_{x} \rho s_{i} u \leq 0, \quad i=1, \ldots, N .
$$

The mass of the Borel measures $\left[\left(p_{i}-\frac{\mu_{i}}{\mu_{N}} p_{N}\right) \partial_{x} u\right]_{\phi}$ in (35) across shock solutions finds a clear definition from (31). Let us conclude this section with the following easy result which proof is left to the reader : 
Corollary 4 Let us consider a piecewise Lipschitz continuous solution $\mathbf{V}$ of (36) (i.e. of (33)). In the zone of smoothness of $\mathbf{V}$, one has:

$$
\partial_{t} \rho \varepsilon_{i}+\partial_{x} \rho \varepsilon_{i} u+p_{i} \partial_{x} u=0, \quad i=1, \ldots, N,
$$

i.e.

$$
\partial_{t} \rho s_{i}+\partial_{x} \rho s_{i} u=0, \quad i=1, \ldots, N,
$$

while shock solutions obey for all $i=1, \ldots, N-1$ :

$$
\begin{gathered}
m\left(\varepsilon_{i}^{+}-\varepsilon_{i}^{-}\right)+\int_{0}^{1} p_{i}\left(\phi\left(s ; \mathbf{V}^{-}, \mathbf{V}^{+}\right)\right) \partial_{s} u\left(\phi\left(s ; \mathbf{V}^{-}, \mathbf{V}^{+}\right)\right) d s= \\
\frac{\mu_{i}}{\mu_{N}}\left\{m\left(\varepsilon_{N}^{+}-\varepsilon_{N}^{-}\right)+\int_{0}^{1} p_{N}\left(\phi\left(s ; \mathbf{V}^{-}, \mathbf{V}^{+}\right)\right) \partial_{s} u\left(\phi\left(s ; \mathbf{V}^{-}, \mathbf{V}^{+}\right)\right) d s\right\},
\end{gathered}
$$

with

$$
m\left(s_{i}^{+}-s_{i}^{-}\right)<0, \quad i=1, \ldots, N .
$$

The identities (38) and (40) are clearly nothing but the extensions of (15) and the corresponding one in (16) respectively, when expressed in the general setting in terms of internal energies instead of entropies. The Rankine-Hugoniot like conditions (40) will be referred in the sequel as to generalized jump conditions.

\section{Exact Riemann Solver and the Generalized Jump Conditions}

We now address the numerical approximation of the weak solutions of (36)-(37). The key issue turns out to enforce at the discrete level each of the $N$ entropy rates of dissipation to be kept in balance according to the ratios of the viscosities while ensuring the discrete conservation of the density, the momentum and the total energy. The system (36) has been precisely introduced to fulfill this requirement. We shall prove below, on the basis of the Godunov method, that the equivalence with the original system (33) is lost at the discrete level. Approximating piecewise Lipschitz continuous solutions via (33) actually results in large errors between discrete and exact solutions. The roots of such a failure will be clearly identified with the numerical dissipation in the method which produces entropy dissipations at the wrong rates. This observation will naturally lead us to enforce the required balance in the entropies dissipation rates at the discrete level. Doing so, we shall end up with a finite volume approximation of the equivalent system (36), which is fully explicit, satisfies several stability properties under a classical CFL condition and produces approximate solutions in close agreement with the exact ones.

The derivation of the method and its properties is performed here on the basis of the Godunov method, and it will find a fairly natural extension in the next section devoted to Approximate Riemann Solvers. Let us first briefly report here some useful properties of the exact Riemann solution for (33) that are in order to prove the forthcoming statements.

Existence (away from vacuum) and uniqueness of a solution of the Riemann problem for (33) has been proved in[7]. As strongly suggested by lemma 1, the Riemann solution is made of at most three simple waves (under the Weyl's assumptions) separating four constant states : namely $\mathbf{V}_{L}$, $\mathbf{V}_{L}^{\star}, \mathbf{V}_{R}^{\star}$ and $\mathbf{V}_{R}$ where with classical notations $\mathbf{V}_{L}$ and $\mathbf{V}_{R}$ denote the two constant states in the Riemann initial data. The Riemann solution, we denote $\mathbf{w}\left(x / t, \mathbf{V}_{L}, \mathbf{V}_{R}\right)$ in the sequel, is shown below to obey maximum principles on each of the specific entropies. This result extends a property proved by Tadmor in the classical Euler setting (i.e. $N=1$ ) and will play an important role hereafter. 
Lemma 5 The self similar solution $\mathbf{w}\left(x / t, \mathbf{V}_{L}, \mathbf{V}_{R}\right)$ of the Cauchy problem (33) with initial data $\mathbf{V}_{0}(x)=\mathbf{V}_{L}$ if $x<0$ and $\mathbf{V}_{R}$ otherwise is such that:

$$
s_{i}\left(., \mathbf{V}_{L}, \mathbf{V}_{R}\right) \leq \max \left(s_{i L}, s_{i R}\right), \quad i=1, \ldots, N .
$$

\section{Proof}

Using standard arguments (see[13]) it can be easily proved that each of the specific entropies $s_{i}$ are Riemann invariants for the first and third genuine non linear fields and jump freely across the contact discontinuity. Consequently, $s_{i}$ can achieve distinct values from $s_{i L}$ and $s_{i R}$ only across shock solutions associated with these two fields and therefore at most two distinct values : namely $s_{i}^{\star}$ and $s_{i}^{\star}$. But across 1-shock solutions, the relative mass flux $m$ in (41) is positive (see[13] in the classical Euler setting) so that $s_{i}^{\star}<s_{i L}$, while across 3 -shock solutions, $m$ is negative so that $s_{i}^{\star}<s_{i R}$. This concludes the proof. Let us now address the numerical approximation of the weak solutions of (36)-(37). For simplicity in the notations, we restrict ourselves to uniform cartesian discretization of $\mathbb{R}_{x} \times \mathbb{R}_{t}^{+}$defined by a constant time step $\Delta t$ and a constant space step $\Delta x$. Introducing $x_{j+1 / 2}=(j+1 / 2) \Delta x$ for $j \in \mathbb{Z}$ and $t^{n}=n \Delta t$ for $n \in N$, the cartesian grids under consideration then read $\cup_{j \in \mathbb{Z}, n \in \mathbb{N}} \mathcal{C}_{j}^{n}$ where $\mathcal{C}_{j}^{n}:=\left[x_{j-1 / 2}, x_{j+1 / 2}\right) \times\left[t^{n}, t^{n+1}\right)$. The approximate solution of the Cauchy problem (33) with $\mathbf{V}_{0}$ as initial data, we denote $\mathbf{V}_{\lambda}(x, t)$ in the sequel with $\lambda=\Delta t / \Delta x$, is classically sought as a piecewise constant function at each time level $t^{n}$,

$$
\mathbf{V}_{\lambda}\left(x, t^{n}\right):=\mathbf{V}_{j}^{n}, \quad \text { for all } x \in\left[x_{j-1 / 2}, x_{j+1 / 2}\right), \quad n>0, \quad j \in \mathbb{Z}
$$

with when $n=0$ :

$$
\mathbf{V}_{j}^{0}=\frac{1}{\Delta x} \int_{x_{j-1 / 2}}^{x_{j+1 / 2}} \mathbf{V}_{0}(x) d x, \text { for all } j \in \mathbb{Z} .
$$

Assuming that the approximate solution $\mathbf{V}_{\lambda}\left(x, t^{n}\right)$ is known at a given time $t^{n} \geq 0$, this one is then defined for $t \in\left[t^{n}, t^{n+1}\right)$ as the solution of the Cauchy problem (33) with $\mathbf{V}_{\lambda}\left(x, t^{n}\right)$ as initial data. Provided that $\Delta t$ is chosen small enough, i.e. under the CFL restriction :

$$
\lambda \max _{\mathbf{V}} \rho(\mathbf{A}(\mathbf{V})) \leq \frac{1}{2}
$$

where the maximum is taken over all the $\mathbf{V}$ under consideration, $\mathbf{V}_{\lambda}(x, t)$ with $t \in\left[t^{n}, t^{n+1}\right)$ is nothing but the juxtaposition of a sequence of non interacting adjacent Riemann solutions $\mathbf{w}((x-$ $\left.\left.x_{j+1 / 2}\right) /\left(t-t^{n}\right) ; \mathbf{V}_{j}^{n}, \mathbf{V}_{j+1}^{n}\right)$, centered at each cell interface $x_{j+1 / 2}$. Let us then consider the $L^{2}$ projection of this solution at time $t^{n+1-}$ onto piecewise constant functions :

$$
\mathbf{V}_{j}^{n+1-}=\frac{1}{\Delta x} \int_{x_{j-1 / 2}}^{x_{j+1 / 2}} \mathbf{V}_{\lambda}\left(x, t^{n+1-}\right) d x, \quad j \in \mathbb{Z}
$$

Notice that the averages (45) can be given the convenient equivalent form :

$$
\left\{\begin{array}{l}
\rho_{j}^{n+1-}=\rho_{j}^{n}-\lambda \Delta\{\rho u\}_{j+1 / 2}^{n}, \\
(\rho u)_{j}^{n+1-}=(\rho u)_{j}^{n}-\lambda \Delta\left\{\rho u^{2}+\sum_{i=N}^{N} p_{i}\right\}_{j+1 / 2}^{n},
\end{array}\right.
$$

while the internal energies $\rho \varepsilon_{i}$ for $i \in\{1, \ldots, N\}$ update according to :

$$
\left(\rho \varepsilon_{i}\right)_{j}^{n+1-}=\left(\rho \varepsilon_{i}\right)_{j}^{n}-\lambda \Delta\left\{\rho \varepsilon_{i} u\right\}_{j+1 / 2}^{n}+\lambda<\left(N_{i}-\left[p_{i} \partial_{x} u\right]_{\phi}\right)\left(\mathbf{V}_{\lambda}(x, t)\right), \mathcal{C}_{j}^{n}>.
$$

In (46), (47) and hereafter, the increment $\Delta\{X\}_{j+1 / 2}^{n}$ equals $X_{j+1 / 2}^{n}-X_{j-1 / 2}^{n}$ where $X_{j+1 / 2}^{n}$ is any numerical flux function entering the discrete problem. These numerical fluxes $X_{j+1 / 2}^{n}$ at the 
cell interface $x_{j+1 / 2}$ classically find the definition $X_{j+1 / 2}^{n}=\{X\}\left(\mathbf{w}\left(0^{+} ; \mathbf{V}_{j}^{n}, \mathbf{V}_{j+1}^{n}\right)\right)$ under the CFL restriction (44) while with clear notations, one has :

$$
\begin{gathered}
<\left(N_{i}-\left[p_{i} \partial_{x} u\right]_{\phi}\right)\left(\mathbf{V}_{\lambda}(x, t)\right), \mathcal{C}_{j}^{n}>= \\
-\iint_{\mathcal{C}_{j}^{n}}\left\{p_{i} \partial_{x} u\right\}\left(\mathbf{V}_{\lambda}(x, t)\right) d x d t+\sum_{\text {shocks } \in \mathcal{C}_{j}^{n}} \rho^{-}\left(u^{-}-\sigma\right)\left(\varepsilon_{i}^{+}-\varepsilon_{i}^{-}\right) .
\end{gathered}
$$

Here the first contribution comes from the fact that the Borel measure $\mathcal{N}_{i}$ in (34) identically vanishes everywhere except at shock locations while the second contribution clearly results from the jump conditions (29) across propagating shocks in $\mathcal{C}_{j}^{n}$.

According to the usual Godunov's procedure, one would update the approximate solution at time $t^{n+1}$ when defining $\mathbf{V}_{\lambda}\left(x, t^{n+1}\right)=\mathbf{V}_{j}^{n+1-}$ for all $x \in\left[x_{j-1 / 2}, x_{j+1 / 2}\right)$ and $j \in \mathbb{Z}$. However, such an updating formula would prevent the $L^{1}$ norm of the total energy to be preserved with time because of the next statement :

Proposition 3 Under the CFL condition (44), the following inequality holds for all $j \in \mathbb{Z}$ :

$$
\{\rho E\}\left(\mathbf{V}_{j}^{n+1-}\right) \leq\{\rho E\}\left(\mathbf{V}_{j}^{n}\right)-\lambda \Delta\{\rho H u\}\left(\mathbf{w}\left(0^{+} ; \mathbf{V}_{j}^{n}, \mathbf{V}_{j+1}^{n}\right)\right),
$$

such an inequality being strict generally speaking.

The proof of this result is postponed at the end of the present section. Actually, it follows from two facts. At first, the weak solutions of (33) are known to satisfy a conservation property on the total energy, so that it can be shown that the right-hand side in (48) coincides with $(\rho E)_{j}^{n+1-}$ defined by

$$
(\rho E)_{j}^{n+1-}:=\frac{1}{\Delta x} \int_{x_{j-1 / 2}}^{x_{j+1 / 2}}\{\rho E\}\left(\mathbf{V}_{\lambda}\left(x, t^{n+1-}\right)\right) d x .
$$

Then, inequality (48) is obtained from (49) when invoking the classical Jensen's inequality together with suitable convexity properties (see proof below for details). More precisely, the following estimate holds (see Coquel and LeFloch [9] for the details)

$$
\{\rho E\}\left(\mathbf{V}_{j}^{n+1-}\right)=(\rho E)_{j}^{n+1-}-\mathcal{O}(1)\left(\left\|\mathbf{V}_{j}^{n}-\mathbf{V}_{j-1}^{n}\right\|^{2}+\left\|\mathbf{V}_{j+1}^{n}-\mathbf{V}_{j}^{n}\right\|^{2}\right),
$$

where $\mathcal{O}(1)$ depends on the convexity modulus of $\{\rho E\}(\mathbf{V})$. Hence, the total energy dramatically decreases across moderate to strong shock solutions. Rephrazing the inequality (48), the formulae (46)-(47) make the $L^{1}$-norm of the total energy to strictly decrease with time and as a consequence, cannot provide us with a relevant numerical method for approximating the discontinuous solutions under consideration. Numerical evidences of such a failure, given in section 6, clearly indicate that the approximate solutions derived from (46)-(47) grossly disagree with the exact ones. One would be therefore tempted to enforce for validity the conservation of the discrete total energy when defining for all $j$ in $\mathbb{Z}$ :

$$
\begin{aligned}
& (\rho E)_{j}^{n+1-}:=\frac{1}{\Delta x} \int_{x_{j-1 / 2}}^{x_{j+1 / 2}}\{\rho E\}\left(\mathbf{V}_{\lambda}\left(x, t^{n+1-}\right)\right) d x \\
& =\{\rho E\}\left(\mathbf{V}_{j}^{n}\right)-\lambda \Delta\{\rho H u\}\left(\mathbf{w}\left(0^{+} ; \mathbf{V}_{j}^{n}, \mathbf{V}_{j+1}^{n}\right)\right),
\end{aligned}
$$

while keeping unchanged the definition of $\rho_{j}^{n+1-},(\rho u)_{j}^{n+1-}$ and up to some renumbering, the first $(N-1)$ internal energies $\left(\rho \varepsilon_{i}\right)_{j}^{n+1-}$. Here, the last internal energy would thus be redefined according to :

$$
\left\{\rho \varepsilon_{N}\right\}\left(\overline{\mathbf{V}}_{j}^{n+1-}\right):=(\rho E)_{j}^{n+1-}-\frac{\left((\rho u)_{j}^{n+1-}\right)^{2}}{2 \rho_{j}^{n+1-}}-\sum_{i=1}^{N-1}\left(\rho \varepsilon_{i}\right)_{j}^{n+1-}
$$


where we have set :

$$
\overline{\mathbf{V}}_{j}^{n+1-}={ }^{T}\left(\rho_{j}^{n+1-},(\rho u)_{j}^{n+1-},(\rho E)_{j}^{n+1-},\left\{\left(\rho \varepsilon_{i}\right)_{j}^{n+1-}\right\}_{1 \leq i \leq N-1}\right) .
$$

Doing so, one would then end up with a failure of (22) and then of the generalized jump conditions (40) at the discrete level as expressed in the next statement (which proof is also postponed at the end of this section) :

Proposition 4 Under the CFL condition (44), the following inequalities hold true for all $i=$ $1, \ldots, N$ and all $j \in \mathbb{Z}$ :

$$
\begin{gathered}
\mu_{N}\left(\left(\rho \varepsilon_{i}\right)_{j}^{n+1-}-\left(\rho \varepsilon_{i}\right)_{j}^{n}+\lambda \Delta\left\{\rho \varepsilon_{i} u\right\}_{j+1 / 2}^{n}+\lambda<\left[p_{i} \partial_{x} u\right]_{\phi}\left(\mathbf{V}_{\lambda}(x, t)\right), \mathcal{C}_{j}^{n}>\right) \\
\leq \\
\mu_{i}\left(\left\{\rho \varepsilon_{N}\right\}\left(\overline{\mathbf{V}}_{j}^{n+1-}\right)-\left(\rho \varepsilon_{N}\right)_{j}^{n}+\lambda \Delta\left\{\rho \varepsilon_{N} u\right\}_{j+1 / 2}^{n}+\lambda<\left[p_{N} \partial_{x} u\right]_{\phi}\left(\mathbf{V}_{\lambda}(x, t)\right), \mathcal{C}_{j}^{n}>\right),
\end{gathered}
$$

these inequalities being strict generally speaking.

Again, the Jensen's inequality is seen hereafter to be responsible for this negative issue. In order to restore the conservation of the total energy at each time level while preserving the validity of the generalized jump conditions, we are therefore led to keep unchanged the updated values of both the density and momentum :

$$
\rho_{j}^{n+1}=\rho_{j}^{n+1-}, \quad(\rho u)_{j}^{n+1}=(\rho u)_{j}^{n+1-}, \quad \text { for all } j \in \mathbb{Z},
$$

while defining the $N$ internal energies $\left(\rho \varepsilon_{i}\right)_{j}^{n+1}$ as the solutions of the following $(N-1)$ relations :

$$
\begin{gathered}
\mu_{N}\left(\left(\rho \varepsilon_{i}\right)_{j}^{n+1}-\left(\rho \varepsilon_{i}\right)_{j}^{n}+\lambda \Delta\left\{\rho \varepsilon_{j} u\right\}_{j+1 / 2}^{n}+\lambda<\left[p_{i} \partial_{x} u\right]_{\phi}\left(\mathbf{V}_{\lambda}(x, t)\right), \mathcal{C}_{j}^{n}>\right) \\
= \\
\mu_{i}\left(\left(\rho \varepsilon_{N}\right)_{j}^{n+1}-\left(\rho \varepsilon_{N}\right)_{j}^{n}+\lambda \Delta\left\{\rho \varepsilon_{N} u\right\}_{j+1 / 2}^{n}+\lambda<\left[p_{N} \partial_{x} u\right]_{\phi}\left(\mathbf{V}_{\lambda}(x, t)\right), \mathcal{C}_{j}^{n}>\right),
\end{gathered}
$$

supplemented with :

$$
(\rho E)_{j}^{n+1}:=(\rho E)_{j}^{n}-\lambda \Delta\{\rho H u\}_{j+1 / 2}^{n}=\frac{\left((\rho u)_{j}^{n+1}\right)^{2}}{2 \rho_{j}^{n+1}}+\sum_{i=1}^{N}\left(\rho \varepsilon_{i}\right)_{j}^{n+1} .
$$

Let us underline that the above $(N-1)$ identities are nothing but the discrete version of $(22)$. Let us further notice that (34) readily implies for each $j \in \mathbb{Z}$ :

$$
\mu_{N}<N_{i}\left(\mathbf{V}_{\lambda}(x, t)\right), \mathcal{C}_{j}^{n}>=\mu_{i}<N_{N}\left(\mathbf{V}_{\lambda}(x, t)\right), \mathcal{C}_{j}^{n}>
$$

so that the $\left\{\left(\rho \varepsilon_{i}\right)_{j}^{n+1}\right\}_{1 \leq i \leq N}$ equivalently solve :

$$
\left\{\begin{array}{l}
\mu_{N}\left(\left(\rho \varepsilon_{i}\right)_{j}^{n+1}-\left(\rho \varepsilon_{i}\right)_{j}^{n+1-}\right)=\mu_{i}\left(\left(\rho \varepsilon_{N}\right)_{j}^{n+1}-\left(\rho \varepsilon_{N}\right)_{j}^{n+1-}\right), 1 \leq i \leq N-1 \\
\sum_{i=1}^{N}\left(\rho \varepsilon_{i}\right)_{j}^{n+1}=(\rho E)_{j}^{n+1}-\frac{\left((\rho u)_{j}^{n+1}\right)^{2}}{2 \rho_{j}^{n+1}} .
\end{array}\right.
$$

As an immediate consequence, we have : 
Proposition 5 The linear system (57) in the unknown $\left\{\left(\rho \varepsilon_{i}\right)_{j}^{n+1}\right\}_{1 \leq i \leq N}$ admits an unique solution explicitly given by :

$$
\left(\rho \varepsilon_{i}\right)_{j}^{n+1}=\left(\rho \varepsilon_{i}\right)_{j}^{n+1-}+\frac{\mu_{i}}{\sum_{l=1}^{N} \mu_{l}}\left((\rho E)_{j}^{n+1}-\frac{\left((\rho u)_{j}^{n+1}\right)^{2}}{2 \rho_{j}^{n+1}}-\sum_{l=1}^{N}\left(\rho \varepsilon_{l}\right)_{j}^{n+1-}\right),
$$

for all $i=1, \ldots, N$.

The last result of this section proves the stability of the scheme.

Theorem 6 Under the CFL condition (44), the numerical method (45)-(54)-(58) preserves the positivity of the internal energies, that is to say $\left(\rho \varepsilon_{i}\right)_{j}^{n} \geq 0$ for $i=1, \ldots, N$ and all $j \in \mathbb{Z}$. In addition, it obeys for $i=1, \ldots, N$ the following entropy inequalities :

$$
\left(\rho s_{i}\right)_{j}^{n+1}:=\left\{\rho s_{i}\right\}\left(\rho_{j}^{n+1},\left(\rho \varepsilon_{i}\right)_{j}^{n+1}\right) \leq\left(\rho s_{i}\right)_{j}^{n}-\lambda \Delta\left\{\rho s_{i} u\right\}\left(\mathbf{w}\left(0^{+} ; \mathbf{V}_{j}^{n}, \mathbf{V}_{j+1}^{n}\right)\right),
$$

and achieves the next maximum principles on the specific entropies :

$$
\left(s_{i}\right)_{j}^{n+1} \leq \max \left(\left(s_{i}\right)_{j-1}^{n},\left(s_{i}\right)_{j}^{n},\left(s_{i}\right)_{j+1}^{n}\right) .
$$

We conclude with the proofs of the results stated in this section. Let us first establish the proposition 3. Proof (Proposition 3)

Arguing about the convexity of the mapping $(\rho, \rho u) \mapsto \frac{(\rho u)^{2}}{2 \rho}$ and using the definition of the averages (45) at time $t^{n+1-}$, the Jensen's inequality immediately implies

$$
\begin{gathered}
\{\rho E\}\left(\mathbf{V}_{j}^{n+1-}\right):=\frac{\left((\rho u)_{j}^{n+1-}\right)^{2}}{2 \rho_{j}^{n+1-}}+\sum_{i=1}^{N}\left(\rho \varepsilon_{i}\right)_{j}^{n+1-} \leq \\
\frac{1}{\Delta x} \int_{x_{j-1 / 2}}^{x_{j+1 / 2}}\left\{\frac{(\rho u)^{2}}{2 \rho}\right\}\left(\mathbf{V}_{\lambda}\left(x, t^{n+1-}\right)\right) d x+\sum_{i=1}^{N}\left(\rho \varepsilon_{i}\right)_{j}^{n+1-}= \\
\frac{1}{\Delta x} \int_{x_{j-1 / 2}}^{x_{j+1 / 2}}\{\rho E\}\left(\mathbf{V}_{\lambda}\left(x, t^{n+1-}\right)\right) d x .
\end{gathered}
$$

Next, weak solutions of the system (33) are known to obey the following conservation law for the total energy :

$$
\partial_{t} \rho E+\partial_{x}\left(\rho E+\sum_{i=1}^{N} p_{i}\right) u=0, \quad \text { in } \mathcal{D}^{\prime} .
$$

Classical arguments then apply to get the desired identity :

$$
\frac{1}{\Delta x} \int_{x_{j-1 / 2}}^{x_{j+1 / 2}}\{\rho E\}\left(\mathbf{V}_{\lambda}\left(x, t^{n+1-}\right)\right) d x=(\rho E)_{j}^{n}-\lambda \Delta\{\rho H u\}_{j+1 / 2}^{n} .
$$

Let us now turn proving the next proposition. Proof (Proposition 4)

First and by construction,

$$
\left(\rho \varepsilon_{i}\right)_{j}^{n+1-}=\frac{1}{\Delta x} \int_{x_{j-1 / 2}}^{x_{j+1 / 2}}\left(\rho \varepsilon_{i}\right)\left(\mathbf{V}_{\lambda}\left(x, t^{n+1-}\right)\right) d x, \quad 1 \leq i \leq N-1
$$


so that (47) apply, that is to say for $i \in\{1, \ldots, N-1\}$ :

$$
\begin{gathered}
\left(\rho \varepsilon_{i}\right)_{j}^{n+1-}-\left(\rho \varepsilon_{i}\right)_{j}^{n}+\lambda \Delta\left\{\rho \varepsilon_{i} u\right\}_{j+1 / 2}^{n}+\lambda<\left[p_{i} \partial_{x} u\right]_{\phi}\left(\mathbf{V}_{\lambda}(x, t)\right), \mathcal{C}_{j}^{n}>= \\
\lambda<N_{i}\left(\mathbf{V}_{\lambda}(x, t)\right), \mathcal{C}_{j}^{n}>.
\end{gathered}
$$

Let us now check that the last internal energy obeys the following inequality :

$$
\left\{\rho \varepsilon_{N}\right\}\left(\overline{\mathbf{V}}_{j}^{n+1-}\right) \geq \frac{1}{\Delta x} \int_{x_{j-1 / 2}}^{x_{j+1 / 2}}\left(\rho \varepsilon_{N}\right)\left(\mathbf{V}_{\lambda}\left(x, t^{n+1-}\right)\right) d x
$$

To that purpose, it suffices to notice that the total energy inequality (48) proved in proposition 3 just recasts :

$$
\frac{\left((\rho u)_{j}^{n+1-}\right)^{2}}{2 \rho_{j}^{n+1-}}+\sum_{i=1}^{N-1}\left(\rho \varepsilon_{i}\right)_{j}^{n+1-}+\frac{1}{\Delta x} \int_{x_{j-1 / 2}}^{x_{j+1 / 2}}\left\{\rho \varepsilon_{N}\right\}\left(\mathbf{V}_{\lambda}\left(x, t^{n+1-}\right)\right) d x \leq(\rho E)_{j}^{n+1-}
$$

that is from the definition (51):

$$
\begin{gathered}
\left\{\rho \varepsilon_{N}\right\}\left(\overline{\mathbf{V}}_{j}^{n+1-}\right):=(\rho E)_{j}^{n+1-}-\frac{\left((\rho u)_{j}^{n+1-}\right)^{2}}{2 \rho_{j}^{n+1-}}-\sum_{i=1}^{N-1}\left(\rho \varepsilon_{i}\right)_{j}^{n+1-} \geq \\
\frac{1}{\Delta x} \int_{x_{j-1 / 2}}^{x_{j+1 / 2}}\left(\rho \varepsilon_{N}\right)\left(\mathbf{V}_{\lambda}\left(x, t^{n+1-}\right)\right) d x
\end{gathered}
$$

This gives the required inequality (65) which exactly rewrites, when considering the formula (47) for $i=N$ :

$$
\begin{gathered}
\left\{\rho \varepsilon_{N}\right\}\left(\overline{\mathbf{V}}_{j}^{n+1-}\right)-\left(\rho \varepsilon_{N}\right)_{j}^{n}+\lambda \Delta\left\{\rho \varepsilon_{N} u\right\}_{j+1 / 2}^{n}+\lambda<\left[p_{N} \partial_{x} u\right]_{\phi}\left(\mathbf{V}_{\lambda}(x, t)\right), \mathcal{C}_{j}^{n}> \\
\geq \lambda<N_{N}\left(\mathbf{V}_{\lambda}(x, t)\right), \mathcal{C}_{j}^{n}>
\end{gathered}
$$

Let us notice that generally speaking, such an inequality is strict. The positivity of the viscosity coefficients $\mu_{i}$ then easily yields the conclusion from (64) and (67), in view of the identities (56). We next establish the theorem 6. Proof (Theorem 6)

Since by construction, the total energy $(\rho E)_{j}^{n+1}$ finds the conservative discrete formulation (55), we immediately infer from the energy inequality proved in proposition 3 :

$$
\{\rho E\}\left(\mathbf{V}_{j}^{n+1-}\right)=\frac{\left((\rho u)_{j}^{n+1-}\right)^{2}}{2 \rho_{j}^{n+1-}}+\sum_{i=1}^{N}\left(\rho \varepsilon_{i}\right)_{j}^{n+1-} \leq(\rho E)_{j}^{n+1} .
$$

As a consequence, invoking the explicit formulae (58) for defining each of the internal energies $\left(\rho \varepsilon_{i}\right)_{j}^{n+1}$, the positivity of the viscosity coefficients easily ensures that :

$$
\left(\rho \varepsilon_{i}\right)_{j}^{n+1} \geq\left(\rho \varepsilon_{i}\right)_{j}^{n+1-} \text { for all } i \in\{1, \ldots, N\} .
$$

But, $\left(\rho \varepsilon_{i}\right)_{j}^{n+1-}$ stays by construction positive. Indeed, this quantity is nothing but the average (see indeed (45)) of the exact internal energy $\left(\rho \varepsilon_{i}\right)$ evaluated on Riemann solutions under the CFL restriction (44). Such solutions stay within the admissible phase space and this gives the required conclusion. 
Regarding the entropy inequalities (59), the weak solutions of (33) obey in view of the previous section :

$$
\partial_{t} \rho s_{i}+\partial_{x} \rho s_{i} u \leq 0, \quad \mathcal{D}^{\prime},
$$

so that using classical arguments, we arrive at :

$$
\frac{1}{\Delta x} \int_{x_{j-1 / 2}}^{x_{j+1 / 2}}\left\{\rho s_{i}\right\}\left(\mathbf{V}_{\lambda}\left(x, t^{n+1-}\right)\right) d x \leq\left(\rho s_{i}\right)_{j}^{n}-\lambda \Delta\left\{\rho s_{i} u\right\}\left(\mathbf{w}\left(0^{+} ; \mathbf{V}_{j}^{n}, \mathbf{V}_{j+1}^{n}\right)\right)
$$

But, by invoking the convexity of the mapping $\left(\rho, \rho \varepsilon_{i}\right) \mapsto\left\{\rho s_{i}\right\}\left(\rho, \rho \varepsilon_{i}\right)$, we get from the Jensen's inequality :

$$
\left\{\rho s_{i}\right\}\left(\rho_{j}^{n+1-},\left(\rho \varepsilon_{i}\right)_{j}^{n+1-}\right) \leq \frac{1}{\Delta x} \int_{x_{j-1 / 2}}^{x_{j+1 / 2}}\left\{\rho s_{i}\right\}\left(\mathbf{V}_{\lambda}\left(x, t^{n+1-}\right)\right) d x .
$$

Now it is sufficient to notice that:

$$
\frac{\partial \rho s_{i}}{\partial \rho \varepsilon_{i}}=-\frac{1}{T_{i}}<0
$$

to get the required conclusion from the inequality (69).

To prove the validity of the maximum principles on the specific entropies, let us first recall from the beginning of this section, that the Riemann solution at each cell interface $x_{j+1 / 2}$ satisfies :

$$
s_{i}\left(\frac{x-x_{j+1 / 2}}{t-t^{n}}, \mathbf{V}_{j}^{n}, \mathbf{V}_{j+1}^{n}\right) \leq \max \left(\left(s_{i}\right)_{j}^{n},\left(s_{i}\right)_{j+1}^{n}\right) .
$$

Equipped with these inequalities valid for all $j \in \mathbb{Z}$ and all $i=1, \ldots, N$, we deduce from the latter considerations that under the CFL condition (44) :

$$
\begin{gathered}
s_{i}\left(\rho_{j}^{n+1},\left(\rho \varepsilon_{i}\right)_{j}^{n+1}\right):=\frac{\left(\rho s_{i}\right)_{j}^{n+1}}{\rho_{j}^{n+1}} \leq \frac{1}{\Delta x} \int_{x_{j-1 / 2}}^{x_{j+1 / 2}} \frac{\left\{\rho s_{i}\right\}\left(\mathbf{V}_{\lambda}\left(x, t^{n+1}-\right)\right.}{\rho_{j}^{n+1}} d x \leq \\
\max \left(\left(s_{i}\right)_{j}^{n+1},\left(s_{i}\right)_{j}^{n},\left(s_{i}\right)_{j+1}^{n}\right) \frac{1}{\Delta x} \int_{x_{j-1 / 2}}^{x_{j+1 / 2}} \frac{\{\rho\}\left(\mathbf{V}_{\lambda}\left(x, t^{n+1-}\right)\right)}{\rho_{j}^{n+1}} d x,
\end{gathered}
$$

arguing about the positivity of the density in each of the exact Riemann solutions. This is nothing but the required conclusion by definition of $\rho_{j}^{n+1}$.

\section{Approximate Riemann Solvers and the Generalized Jump Con- ditions}

This section aims at generalizing the method described in the previous section when considering Approximate Riemann Solvers. The basic motivation here stems from the lack of a precise knowledge of the required kinetic relations for general pressure and viscosity laws. The extension we propose primarily intends to take advantage of the celebrated framework proposed by Harten, Lax and Van Leer[14]. This framework have indeed received a considerable attention over the past two decades and several valuable methods, ranging from kinetic solvers[19],[13] to Approximate Riemann Solvers with a large variety of intermediate states[21],[14],[12] enter in (see also[4] and the references therein). Our purpose is to directly inherit from all these suitable methods. We show here that most of them may naturally apply to approximate the solutions of (2), despites that they were primarily developed in the setting of systems of (pure) conservation laws. Indeed, these methods are seen in this section to naturally and easily extend to the present setting when enforcing for validity at the discrete level the equalities (22) and then the generalized jump conditions (40) 
while preserving each of their respective stability and precision properties.

We first give a rough description of the extension and then define suitable Approximate Riemann Solvers. With the notations introduced in the first section, let us consider for some given rescaling parameter $\epsilon>0$ the following viscosity form of the system under study :

$$
\partial_{t} \mathbf{U}^{\epsilon}+\partial_{x} \mathbf{F}\left(\mathbf{U}^{\epsilon}\right)=\epsilon \mathcal{R}\left(\mathbf{U}^{\epsilon}, \partial_{x}\left(\mathcal{C}\left(\mathbf{U}^{\epsilon}\right) \partial_{x} \mathbf{U}^{\epsilon}\right)\right),
$$

where $\mathbf{U}$ has been defined in (18), so that the smooth solutions of (73) obey the following additional conservation law :

$$
\partial_{t}\{\rho E\}\left(\mathbf{U}^{\epsilon}\right)+\partial_{x}\left(\rho E^{\epsilon}+\sum_{i=1}^{N} p_{i}^{\epsilon}\right) u^{\epsilon}=\epsilon \partial_{x}\left(\sum_{i=1}^{N} \mu_{i} u^{\epsilon} \partial_{x} u^{\epsilon}\right) .
$$

Let be given $\mathbf{U}_{h}\left(x, t^{n}\right)$ some approximate solution of (73) at time $t^{n}$. We propose to evolve it to the next time level $t^{n+1}$ into two steps that are classically based on a splitting of operators, except for the equation of the total energy which requires a special treatment explained hereafter.

Step 1 The first order (convective) operator, extracted from (73), is solved for small times $t \in[0, \Delta t]$ with $\mathbf{U}_{h}\left(x, t^{n}\right)$ as initial data, that is :

$$
\left\{\begin{array}{l}
\partial_{t} \mathbf{U}+\partial_{x} \mathbf{F}(\mathbf{U})=0 \\
\mathbf{U}(x, 0)=\mathbf{U}_{h}\left(x, t^{n}\right)
\end{array}\right.
$$

Note that for uniqueness of weak solutions, the above Cauchy problem must be supplemented with an entropy selection principle we express here thanks to the total energy (indeed strictly convex in the variable $\mathbf{U})$ :

$$
\partial_{t}\{\rho E\}(\mathbf{U})+\partial_{x}\left(\rho E+\sum_{i=1}^{N} p_{i}\right) u \leq 0 .
$$

Obviously, solving the Cauchy problem (75)-(76) will be precisely the matter of classical Approximate Riemann Solvers.

Step 2 Let $\mathbf{U}_{h}\left(x, t^{n+1-}\right)$ denote the solution of (75)-(76) at time $\Delta t$. This one now naturally serves as initial data to solve the second order (viscosity) operator in (73) for times $t \in[0, \Delta t]$ :

$$
\left\{\begin{array}{l}
\partial_{t} \mathbf{U}^{\epsilon}=\epsilon \mathcal{R}\left(\mathbf{U}^{\epsilon}, \partial_{x}\left(\mathcal{C}\left(\mathbf{U}^{\epsilon}\right) \partial_{x} \mathbf{U}^{\epsilon}\right)\right) \\
\mathbf{U}^{\epsilon}(x, 0)=\mathbf{U}_{h}\left(x, t^{n+1-}\right)
\end{array}\right.
$$

Note that in this second step the total energy evolves according to

$$
\partial_{t}\{\rho E\}\left(\mathbf{U}^{\epsilon}\right)=\epsilon \partial_{x}\left(\sum_{i=1}^{N} \mu_{i} u^{\epsilon} \partial_{x} u^{\epsilon}\right) .
$$

Let us now recast these $(N+2)$ equations of $(77)$ in the $\mathbf{V}$ variable :

$$
\left\{\begin{array}{l}
\partial_{t} \rho^{\epsilon}=0, \\
\partial_{t} \rho u^{\epsilon}=\epsilon \partial_{x}\left(\sum_{i} \mu_{i} \partial_{x} u^{\epsilon}\right), \\
\partial_{t} \rho \varepsilon_{i}^{\epsilon}=\epsilon \mu_{i}\left(\partial_{x} u^{\epsilon}\right)^{2}, \quad \text { for } i=1, \ldots, N,
\end{array}\right.
$$


so that for any given $\epsilon>0$, we infer the following $(N+1)$ equations :

$$
\left\{\begin{array}{l}
\partial_{t} \rho^{\epsilon}=0, \\
\partial_{t} \rho u^{\epsilon}=\epsilon \partial_{x}\left(\sum_{i} \mu_{i} \partial_{x} u^{\epsilon}\right), \\
\mu_{N} \partial_{t} \rho \varepsilon_{i}^{\epsilon}=\mu_{i} \partial_{t} \rho \varepsilon_{N}^{\epsilon}, \quad \text { for } i=1, \ldots, N-1,
\end{array}\right.
$$

which should be supplemented with

$$
\partial_{t}\{\rho E\}\left(\rho^{\epsilon}, \rho u^{\epsilon},\left\{\rho \varepsilon_{i}^{\epsilon}\right\}_{1 \leq i \leq N}\right)=\epsilon \partial_{x}\left(\sum_{i} \mu_{i} u^{\epsilon} \partial_{x} u^{\epsilon}\right) .
$$

Since we do not want to resolve the small scales in $\epsilon$, we consider (79)-(80) in the limit $\epsilon \rightarrow 0$ to get the final form of the problem to be solved in this second step :

$$
\left\{\begin{array}{l}
\partial_{t} \rho=0 \\
\partial_{t} \rho u=0, \\
\mu_{N} \partial_{t} \rho \varepsilon_{i}=\mu_{i} \partial_{t} \rho \varepsilon_{N}, \quad \text { for } i=1, \ldots, N-1,
\end{array}\right.
$$

which once again should be closed by

$$
\partial_{t}\{\rho E\}\left(\rho, \rho u,\left\{\rho \varepsilon_{i}\right\}_{1 \leq i \leq N}\right)=0 .
$$

With the benefit of the vanishing parameter $\epsilon>0$, note that the corresponding solution in (81) is explicitly given by :

$$
\begin{gathered}
\rho(x, t)=\rho_{h}\left(x, t^{n+1-}\right), \quad \rho u(x, t)=(\rho u)_{h}\left(x, t^{n+1-}\right) \\
\rho \varepsilon_{i}(x, t)=\left(\rho \varepsilon_{i}\right)_{h}\left(x, t^{n+1-}\right)+\frac{\mu_{i}}{\mu_{N}}\left(\rho \varepsilon_{N}(x, t)-\left(\rho \varepsilon_{N}\right)_{h}\left(x, t^{n+1-}\right)\right) .
\end{gathered}
$$

Let us underline the very similarity of these identities with the discrete versions derived within the frame of the exact Riemann solver (see (57) for instance).

The total energy should read

$$
\rho E(x, t)=(\rho E)_{h}\left(x, t^{n+1-}\right)
$$

in view of (82). But it has strictly decreased in the first step : see indeed (76). Just like in the previous section, we are thus urged to do abandon this formula so as to restore the conservation of the total energy while enforcing for validity the generalized jump conditions (40). This observation stays at the very basis of the extension we now describe in its details.

Let us first introduce some notations while briefly stating some classical assumptions (see[14],[12]) about approximate Riemann solutions for (75)-(76), which will be of constant use in this section.

\section{Approximate Riemann Solvers for (75)-(76)}

Let be given a family $\mathbf{w}: \mathbb{R} \times \Omega^{2} \rightarrow \Omega$ of Lipschitz continuous functions in the self-similar variable $\xi=x / t$. This family, intending to serve as approximate Riemann solutions for (75)-(76), is subject to the following four consistency properties :

$\left(H_{1}\right) \mathbf{w}(., \mathbf{U}, \mathbf{U})=\mathbf{U}$, for all $\mathbf{U} \in \Omega$.

$\left(H_{2}\right)$ For all bounded subset $\mathcal{B} \in \Omega$, there exists a constant $C$ such that for all pairs $\left(\mathbf{U}_{L}^{1}, \mathbf{U}_{R}^{1}\right)$ and $\left(\mathbf{U}_{L}^{2}, \mathbf{U}_{R}^{2}\right)$ in $\mathcal{B}$,

$$
\left\|\mathbf{w}\left(., \mathbf{U}_{L}^{1}, \mathbf{U}_{R}^{1}\right)-\mathbf{w}\left(., \mathbf{U}_{L}^{2}, \mathbf{U}_{R}^{2}\right)\right\| \leq C\left(\left\|\mathbf{U}_{L}^{2}-\mathbf{U}_{L}^{1}\right\|+\left\|\mathbf{U}_{R}^{2}-\mathbf{U}_{R}^{1}\right\|\right) .
$$


$\left(H_{3}\right)$ There exists an unique smaller and non negative real $\sigma_{m}\left(\mathbf{U}_{L}, \mathbf{U}_{R}\right)$ (the maximum speed at which propagate approximate waves) such that

$$
\left\{\begin{array}{l}
\mathbf{w}\left(\xi, \mathbf{U}_{L}, \mathbf{U}_{R}\right)=\mathbf{U}_{L} \quad \text { if } \quad \xi<-\sigma_{m}\left(\mathbf{U}_{L}, \mathbf{U}_{R}\right) \\
\mathbf{w}\left(\xi, \mathbf{U}_{L}, \mathbf{U}_{R}\right)=\mathbf{U}_{R} \quad \text { if } \quad \xi>\sigma_{m}\left(\mathbf{U}_{L}, \mathbf{U}_{R}\right)
\end{array}\right.
$$

$\left(H_{4}\right)$ For all $\lambda$ satisfying the CFL condition

$$
\lambda \sigma_{m}\left(\mathbf{U}_{L}, \mathbf{U}_{R}\right) \leq \frac{1}{2}
$$

the following average conservation property on $\mathbf{w}$ holds true :

$$
\int_{-1 / 2 \lambda}^{1 / 2 \lambda} \mathbf{w}\left(\xi, \mathbf{U}_{L}, \mathbf{U}_{R}\right) d \xi=\frac{1}{2 \lambda}\left(\mathbf{U}_{L}+\mathbf{U}_{R}\right)-\left(\mathbf{F}\left(\mathbf{U}_{R}\right)-\mathbf{F}\left(\mathbf{U}_{L}\right)\right) .
$$

Equipped with this family of approximate Riemann solutions, let us then consider after Harten, Lax and Van Leer[14] the following two averages :

$$
\begin{aligned}
& \overline{\mathbf{U}}_{L}:=\overline{\mathbf{U}}_{L}\left(\lambda ; \mathbf{U}_{L}, \mathbf{U}_{R}\right)=2 \lambda \int_{-1 / 2 \lambda}^{0} \mathbf{w}\left(\xi, \mathbf{U}_{L}, \mathbf{U}_{R}\right) d \xi, \\
& \overline{\mathbf{U}}_{R}:=\overline{\mathbf{U}}_{R}\left(\lambda ; \mathbf{U}_{L}, \mathbf{U}_{R}\right)=2 \lambda \int_{0}^{1 / 2 \lambda} \mathbf{w}\left(\xi, \mathbf{U}_{L}, \mathbf{U}_{R}\right) d \xi,
\end{aligned}
$$

for any given pair $\left(\mathbf{U}_{L}, \mathbf{U}_{R}\right)$ in $\Omega^{2}$.

\section{Step 1 in details}

Let now be given a piecewise constant approximate solution $\mathbf{U}_{\lambda}\left(x, t^{n}\right)$ for (75). The classical approach for updating this solution at the next time level $t^{n}+\Delta t$ would result, in full agreement with[14], when considering :

$$
\mathbf{U}_{\lambda}\left(x, t^{n}+\Delta t\right)=\frac{1}{2}\left(\overline{\mathbf{U}}_{L}\left(\lambda ; \mathbf{U}_{j}^{n}, \mathbf{U}_{j+1}^{n}\right)+\overline{\mathbf{U}}_{R}\left(\lambda ; \mathbf{U}_{j-1}^{n}, \mathbf{U}_{j}^{n}\right)\right), x \in\left(x_{j-1 / 2}, x_{j+1 / 2}\right),
$$

under the CFL like condition

$$
\lambda \sigma_{m}\left(\mathbf{U}_{j}^{n}, \mathbf{U}_{j+1}^{n}\right) \leq \frac{1}{2}, \quad \lambda=\frac{\Delta t}{\Delta x} .
$$

Here we propose to keep the definition of $\rho_{\lambda}\left(x, t^{n}+\Delta t\right)$ and $(\rho u)_{\lambda}\left(x, t^{n}+\Delta t\right)$ according to (86), that is

$$
\rho_{j}^{n+1-}=\rho_{\lambda}\left(x, t^{n}+\Delta t\right), \quad(\rho u)_{j}^{n+1-}=(\rho u)_{\lambda}\left(x, t^{n}+\Delta t\right),
$$

but to average each of the internal energies $\rho \varepsilon_{i}$ with $i \in\{1, \ldots, N\}$ instead of the corresponding entropy $\rho s_{i}$. Namely, we introduce in full symmetry with (85)-(86) and for all $i=1, \ldots, N$,

$$
\left(\rho \varepsilon_{i}\right)_{j}^{n+1-}=\frac{1}{2}\left({\overline{\rho \varepsilon_{i}}}_{L}\left(\lambda ; \mathbf{U}_{j}^{n}, \mathbf{U}_{j+1}^{n}\right)+{\overline{\rho \varepsilon_{i}}}_{R}\left(\lambda ; \mathbf{U}_{j-1}^{n}, \mathbf{U}_{j}^{n}\right)\right), \quad \text { for all } j \in \mathbb{Z},
$$

where we have set :

$$
\begin{aligned}
& {\overline{\rho \varepsilon_{i}}}_{L}={\overline{\rho \varepsilon_{i}}}_{L}\left(\lambda ; \mathbf{U}_{L}, \mathbf{U}_{R}\right)=2 \lambda \int_{-1 / 2 \lambda}^{0}\left\{\rho \varepsilon_{i}\right\}\left(\mathbf{w}\left(\xi, \mathbf{U}_{L}, \mathbf{U}_{R}\right)\right) d \xi, \\
& \overline{\rho \varepsilon_{i}}={\overline{\rho \varepsilon_{i}}}_{R}\left(\lambda ; \mathbf{U}_{L}, \mathbf{U}_{R}\right)=2 \lambda \int_{0}^{1 / 2 \lambda}\left\{\rho \varepsilon_{i}\right\}\left(\mathbf{w}\left(\xi, \mathbf{U}_{L}, \mathbf{U}_{R}\right)\right) d \xi .
\end{aligned}
$$


Notice that as a consequence of the proposed averages, the entropy $\rho s_{i}$ for $i \in\{1, \ldots, N\}$ actually advances in time according to

$$
\left(\rho s_{i}\right)_{j}^{n+1-}:=\left\{\rho s_{i}\right\}\left(\rho_{j}^{n+1-},\left\{\rho \varepsilon_{i}\right\}_{j}^{n+1-}\right) \leq \frac{1}{2}\left(\overline{\rho s_{i}}+\overline{\rho s_{i}}\right),
$$

by Jensen's inequality. So, (88) and (89) yield the final formula for defining $\mathbf{U}_{j}^{n+1-}$ at the end of the first step. The updating procedure we propose is thus distinct from the usual one and aims at generalizing the approach developed in the previous section (see indeed formula (45) and remark 1 below). But let us stress at this stage that our approach preserves discrete energy inequalities (see the selection principle (76)) as soon as there are satisfied using the standard averages (86). Before addressing this issue, let us recall that the weak consistency property $\left(H_{4}\right)$ is well known to give birth to some mapping $\mathcal{F}: \Omega^{2} \rightarrow \mathbb{R}^{N+2}$ so that (85) by construction recasts in

$$
\begin{aligned}
& \overline{\mathbf{U}}_{R}\left(\lambda ; \mathbf{U}_{L}, \mathbf{U}_{R}\right)=\mathbf{U}_{R}-2 \lambda\left(\mathbf{F}\left(\mathbf{U}_{R}\right)-\mathcal{F}\left(\mathbf{U}_{L}, \mathbf{U}_{R}\right)\right), \\
& \overline{\mathbf{U}}_{L}\left(\lambda ; \mathbf{U}_{L}, \mathbf{U}_{R}\right)=\mathbf{U}_{L}-2 \lambda\left(\mathcal{F}\left(\mathbf{U}_{L}, \mathbf{U}_{R}\right)-\mathbf{F}\left(\mathbf{U}_{L}\right)\right) .
\end{aligned}
$$

Each of these two identities equivalently defines the so-called numerical flux function $\mathcal{F}$ which is easily seen from $\left(H_{1}\right)-\left(H_{2}\right)$ to be consistent in the usual sense of the Lax-Wendroff theorem (see[12]) and with the notations of the last section. Thus and as a consequence of (88)-(92), both the density and the momentum find an expected conservative updating formula

$$
\begin{gathered}
\rho_{j}^{n+1-}=\rho_{j}^{n}-\lambda\left(\mathcal{F}_{\rho}\left(\mathbf{U}_{j}^{n}, \mathbf{U}_{j+1}^{n}\right)-\mathcal{F}_{\rho}\left(\mathbf{U}_{j-1}^{n}, \mathbf{U}_{j}^{n}\right)\right), \\
(\rho u)_{j}^{n+1-}=(\rho u)_{j}^{n}-\lambda\left(\mathcal{F}_{\rho u}\left(\mathbf{U}_{j}^{n}, \mathbf{U}_{j+1}^{n}\right)-\mathcal{F}_{\rho u}\left(\mathbf{U}_{j-1}^{n}, \mathbf{U}_{j}^{n}\right)\right),
\end{gathered}
$$

where clear notations have been used. Such a conclusion obviously fails for the internal energies since their governing equations are not in conservation form. It is however expected that the averages (89) actually yield some relevant discrete forms of the associated partial differential equations. In the case of the exact Riemann solver but for the pure conservative system (75)-(76), we indeed get with clear notations :

$$
\left(\rho \varepsilon_{i}\right)_{j}^{n+1-}=\left(\rho \varepsilon_{i}\right)_{j}^{n}-\lambda \Delta\left(\rho \varepsilon_{i} u\right)_{j+1 / 2}^{n}-\lambda<p_{i} \partial_{x} u, \mathcal{C}_{j}^{n}>.
$$

Remark 1

By contrast with (93), adopting the usual procedure for advancing in time the discrete solution, i.e. averaging each of the entropies $\rho s_{i}$ for $i \in\{1, \ldots, N\}$, would have yield, within the setting of the exact Riemann solver,

$$
\left\{\rho \varepsilon_{i}\right\}\left(\rho_{j}^{n+1-},\left(\rho s_{i}\right)_{j}^{n+1-}\right) \neq\left(\rho \varepsilon_{i}\right)_{j}^{n}-\lambda \Delta\left(\rho \varepsilon_{i} u\right)_{j+1 / 2}^{n}-\lambda<p_{i} \partial_{x} u, \mathcal{C}_{j}^{n}>
$$

because of the Jensen's inequality. This apparent lack of consistency with the underlying partial differential equations therefore strongly promotes the updating strategy we have put forward in this section.

Without further details about the family of approximate Riemann solutions, it seems rather untractable to give an equivalent expression form for the averages (89). Let us however underline that such averages can be actually easily evaluated when considering the hierarchy of Approximate Riemann Solvers with $k$ intermediate states $(0<k<N+1)$ separated by discontinuities. We refer the reader to[6] for a suitable example. 
Let us now considering the existence of in-cell energy inequalities for our procedure. After Harten, Lax and Van Leer, we classically now ask the family of approximate Riemann solutions to obey the following weak consistency property with the Lax inequality (76) :

$\left(H_{5}\right)$ For all $\lambda$ satisfying the CFL condition (83), the following average dissipation property on $\{\rho E\}(\mathbf{w})$ holds true :

$$
\begin{gathered}
\int_{-1 / 2 \lambda}^{1 / 2 \lambda}\{\rho E\}\left(\mathbf{w}\left(\xi, \mathbf{U}_{L}, \mathbf{U}_{R}\right)\right) d \xi \leq \\
\frac{1}{2 \lambda}\left(\{\rho E\}\left(\mathbf{U}_{L}\right)+\{\rho E\}\left(\mathbf{U}_{R}\right)\right)-\left(\{\rho H u\}\left(\mathbf{U}_{R}\right)-\{\rho H u\}\left(\mathbf{U}_{L}\right)\right) .
\end{gathered}
$$

Then, defining

$$
\begin{aligned}
& {\overline{\{\rho E\}_{L}}}_{L}={\overline{\{\rho E\}_{L}}}_{L}\left(\lambda ; \mathbf{U}_{L}, \mathbf{U}_{R}\right)=2 \lambda \int_{-1 / 2 \lambda}^{0}\{\rho E\}\left(\mathbf{w}\left(\xi, \mathbf{U}_{L}, \mathbf{U}_{R}\right)\right) d \xi \\
& {\overline{\{\rho E\}_{R}}}={\overline{\{\rho E\}_{R}}}_{R}\left(\lambda ; \mathbf{U}_{L}, \mathbf{U}_{R}\right)=2 \lambda \int_{0}^{1 / 2 \lambda}\{\rho E\}\left(\mathbf{w}\left(\xi, \mathbf{U}_{L}, \mathbf{U}_{R}\right)\right) d \xi
\end{aligned}
$$

a direct consequence of the above consistency property is the expected easy following result (see[12] page 153).

Lemma 7 Let $\{\rho \mathcal{H} u\}^{-}$and $\{\rho \mathcal{H} u\}^{+}$be two $\Omega^{2} \rightarrow \mathbb{R}$ mappings defined by :

$$
\begin{aligned}
\{\rho \mathcal{H} u\}^{-}\left(\mathbf{U}_{L}, \mathbf{U}_{R}\right) & =\{\rho H u\}\left(\mathbf{U}_{R}\right)+\frac{1}{2 \lambda}\left({\overline{\{\rho E\}_{R}}}_{R}\left(\lambda ; \mathbf{U}_{L}, \mathbf{U}_{R}\right)-\{\rho E\}\left(\mathbf{U}_{R}\right)\right), \\
\{\rho \mathcal{H} u\}^{+}\left(\mathbf{U}_{L}, \mathbf{U}_{R}\right) & =\{\rho H u\}\left(\mathbf{U}_{L}\right)-\frac{1}{2 \lambda}\left(\overline{\{\rho E\}_{L}}\left(\lambda_{L} ; \mathbf{U}_{L}, \mathbf{U}_{R}\right)-\{\rho E\}\left(\mathbf{U}_{L}\right)\right),
\end{aligned}
$$

for all $\left(\mathbf{U}_{L}, \mathbf{U}_{R}\right) \in \Omega^{2}$.

Assume that the CFL condition (83) and hypothesis $(H)$ are valid.

Then, we have for all $\left(\mathbf{U}, \mathbf{U}_{L}, \mathbf{U}_{R}\right) \in \Omega^{3}$ :

$$
\{\rho \mathcal{H} u\}^{-}\left(\mathbf{U}_{L}, \mathbf{U}_{R}\right) \leq\{\rho \mathcal{H} u\}^{+}\left(\mathbf{U}_{L}, \mathbf{U}_{R}\right),
$$

and

$$
\{\rho \mathcal{H} u\}^{-}(\mathbf{U}, \mathbf{U})=\{\rho \mathcal{H} u\}^{+}(\mathbf{U}, \mathbf{U})=\{\rho H u\}(\mathbf{U}) .
$$

Let us then state the main result of the step 1.

Proposition 6 Let the approximate solution be advanced according to (88)-(91) under the CFL restriction (87). Then provided that the entropy consistency condition $\left(H_{5}\right)$ is met, the following in-cell energy inequalities are satisfied for all $j \in \mathbb{Z}$ :

$$
\{\rho E\}\left(\mathbf{U}_{j}^{n+1-}\right)-(\rho E)_{j}^{n}+\lambda \Delta\{\rho \mathcal{H} u\}^{+}\left(\mathbf{U}_{j}^{n}, \mathbf{U}_{j+1}^{n}\right) \leq 0 .
$$

In addition, we have :

$$
\left(\rho s_{i}\right)_{j}^{n+1-}-\left(\rho s_{i}\right)_{j}^{n}+\lambda \Delta\left\{\rho s_{i} u\right\}_{j+1 / 2}^{n} \leq 0,
$$

for all $i \in\{1, \ldots, N\}$.

The proof is postponed at the end of the present section. It will clearly indicate that energy inequalities identical to (97) but with the energy numerical flux $\{\rho \mathcal{H} u\}^{-}$also hold. For definiteness in the forthcoming developments, we choose without restriction the flux $\{\rho \mathcal{H} u\}^{+}$. Observe in addition that these energy inequalities are in complete analogy with the ones obtained in the setting of the 
exact Riemann solver (see proposition 3).

Restoring the conservation of the total energy while enforcing for validity the generalized jump conditions is now the aim of the second step in the method.

\section{Step 2 in details}

Following the guidelines of the rough description of the method, we are led to set :

$$
\rho_{j}^{n+1}=\rho_{j}^{n+1-}, \quad(\rho u)_{j}^{n+1}=(\rho u)_{j}^{n+1-}, \quad \text { for all } j \in \mathbb{Z},
$$

while defining the $N$ internal energies $\left(\rho \varepsilon_{i}\right)_{j}^{n+1}$ as the solutions of the following linear system :

$$
\left\{\begin{array}{l}
\mu_{N}\left(\left(\rho \varepsilon_{i}\right)_{j}^{n+1}-\left(\rho \varepsilon_{i}\right)_{j}^{n+1-}\right)=\mu_{i}\left(\left(\rho \varepsilon_{N}\right)_{j}^{n+1}-\left(\rho \varepsilon_{N}\right)_{j}^{n+1-}\right), 1 \leq i \leq N-1, \\
\sum_{i=1}^{N}\left(\rho \varepsilon_{i}\right)_{j}^{n+1}=(\rho E)_{j}^{n+1}-\frac{\left((\rho u)_{j}^{n+1}\right)^{2}}{2 \rho_{j}^{n+1}} .
\end{array}\right.
$$

In order to restore the conservation of the total energy, we propose to advance the total energy $(\rho E)_{j}^{n+1}$ in time according to :

$$
(\rho E)_{j}^{n+1}:=(\rho E)_{j}^{n}-\lambda \Delta\{\rho \mathcal{H} u\}^{+}\left(\mathbf{U}_{j}^{n}, \mathbf{U}_{j+1}^{n}\right) .
$$

As a consequence of these definitions we have :

Proposition 7 The linear system (100) in the unknown $\left\{\left(\rho \varepsilon_{i}\right)_{j}^{n+1}\right\}_{1 \leq i \leq N}$ admits an unique solution explicitly given by :

$$
\left(\rho \varepsilon_{i}\right)_{j}^{n+1}=\left(\rho \varepsilon_{i}\right)_{j}^{n+1-}+\frac{\mu_{i}}{\sum_{l=1}^{N} \mu_{l}}\left((\rho E)_{j}^{n+1}-\frac{\left((\rho u)_{j}^{n+1}\right)^{2}}{2 \rho_{j}^{n+1}}-\sum_{l=1}^{N}\left(\rho \varepsilon_{l}\right)_{j}^{n+1-}\right),
$$

for all $i=1, \ldots, N$.

This statement highlights the complete symmetry in the updating formulae coming either with the exact Riemann solver considered in the previous section or with the extensions we propose (see proposition 5).

Remark 2

As soon as the Approximate Riemann Solver under consideration allows for a discrete form of the partial differential equations governing $\left\{\rho \varepsilon_{i}\right\}_{i=1, \ldots, N}$ (see remark 1), then (100) reads

$$
\begin{gathered}
\mu_{N}\left(\left(\rho \varepsilon_{i}\right)_{j}^{n+1}-\left(\rho \varepsilon_{i}\right)_{j}^{n}+\lambda \Delta\left\{\rho \varepsilon_{j} u\right\}_{j+1 / 2}^{n}+\lambda<p_{i} \partial_{x} u, \mathcal{C}_{j}^{n}>\right) \\
= \\
\mu_{i}\left(\left(\rho \varepsilon_{N}\right)_{j}^{n+1}-\left(\rho \varepsilon_{N}\right)_{j}^{n}+\lambda \Delta\left\{\rho \varepsilon_{N} u\right\}_{j+1 / 2}^{n}+\lambda<p_{N} \partial_{x} u, \mathcal{C}_{j}^{n}>\right),
\end{gathered}
$$

which is nothing but a discrete version of (22).

In addition, these extensions share with the exact Riemann solver the following desirable stability properties : 
Theorem 8 Under the CFL condition (87), the numerical method (88)-(89)-(99)-(101)-(102) preserves the positivity of the internal energies, that is to say $\left(\rho \varepsilon_{i}\right)_{j}^{n+1} \geq 0$ for $i=1, \ldots, N$ and all $j \in \mathbb{Z}$. Moreover, it obeys for $i=1, \ldots, N$ and all $j \in \mathbb{Z}$ the following entropy inequalities :

$$
\left(\rho s_{i}\right)_{j}^{n+1}:=\left\{\rho s_{i}\right\}\left(\rho_{j}^{n+1},\left(\rho \varepsilon_{i}\right)_{j}^{n+1}\right) \leq\left(\rho s_{i}\right)_{j}^{n}-\lambda \Delta\left\{\rho s_{i} u\right\}\left(\mathbf{U}_{j}^{n}, \mathbf{U}_{j+1}^{n}\right) .
$$

Assuming in addition that the family of approximate Riemann solutions under consideration obeys the following weak form of the maximum principles on the specific entropies $\left\{s_{i}\right\}_{i=1, \ldots, N}$ :

$$
\begin{aligned}
\int_{-1 / 2 \lambda}^{0}\left\{\rho s_{i}\right\}\left(\mathbf{w}\left(\xi, \mathbf{U}_{L}, \mathbf{U}_{R}\right)\right) d \xi & \leq \max \left(\left(s_{i}\right)_{L},\left(s_{i}\right)_{R}\right) \int_{-1 / 2 \lambda}^{0}\{\rho\}\left(\mathbf{w}\left(\xi, \mathbf{U}_{L}, \mathbf{U}_{R}\right)\right) d \xi, \\
\int_{0}^{1 / 2 \lambda}\left\{\rho s_{i}\right\}\left(\mathbf{w}\left(\xi, \mathbf{U}_{L}, \mathbf{U}_{R}\right)\right) d \xi & \leq \max \left(\left(s_{i}\right)_{L},\left(s_{i}\right)_{R}\right) \int_{0}^{1 / 2 \lambda}\{\rho\}\left(\mathbf{w}\left(\xi, \mathbf{U}_{L}, \mathbf{U}_{R}\right)\right) d \xi,
\end{aligned}
$$

then we have for all $i=1, \ldots, N$ :

$$
\left(s_{i}\right)_{j}^{n+1} \leq \max \left(\left(s_{i}\right)_{j-1}^{n},\left(s_{i}\right)_{j}^{n},\left(s_{i}\right)_{j+1}^{n}\right), \quad j \in \mathbb{Z} .
$$

We conclude this section when giving the proofs of the results we have claimed true. To that purpose, we previously state and prove the following lemma.

Lemma 9 Assume that the Approximate Riemann Solver $\mathbf{w}$ obeys (104). Then, with clear notations, the entropy inequalities :

$$
\begin{gathered}
\left\{\rho s_{i}\right\}\left(\bar{\rho}_{L},{\overline{\rho \varepsilon_{i}}}_{L}\right) \leq\left\{\rho s_{i}\right\}\left(\rho_{L},\left(\rho \varepsilon_{i}\right)_{L}\right)-2 \lambda\left(\mathcal{F}_{\rho s_{i}}\left(\mathbf{U}_{L}, \mathbf{U}_{R}\right)-\left(\rho s_{i} u\right)_{L}\right), \\
\left\{\rho s_{i}\right\}\left(\bar{\rho}_{R}, \overline{\rho \varepsilon_{i}}\right) \leq\left\{\rho s_{i}\right\}\left(\rho_{R},\left(\rho \varepsilon_{i}\right)_{R}\right)-2 \lambda\left(\left(\rho s_{i} u\right)_{R}-\mathcal{F}_{\rho s_{i}}\left(\mathbf{U}_{L}, \mathbf{U}_{R}\right)\right),
\end{gathered}
$$

hold true for all $i=1, \ldots, N$, as well as the maximum principles :

$$
\begin{aligned}
& \left\{s_{i}\right\}\left(\bar{\rho}_{L},{\overline{\rho \varepsilon_{i}}}_{L}\right):=\frac{\left\{\rho s_{i}\right\}\left(\bar{\rho}_{L}, \overline{\rho \varepsilon_{i}}\right)}{\bar{\rho}_{L}} \leq \max \left(\left(s_{i}\right)_{L},\left(s_{i}\right)_{R}\right), \\
& \left\{s_{i}\right\}\left(\bar{\rho}_{R},{\overline{\rho \varepsilon_{i}}}_{R}\right):=\frac{\left\{\rho s_{i}\right\}\left(\bar{\rho}_{R}, \overline{\rho \varepsilon_{i}}\right)}{\bar{\rho}_{R}} \leq \max \left(\left(s_{i}\right)_{L},\left(s_{i}\right)_{R}\right) .
\end{aligned}
$$

Proof (Lemma 9)

First, arguing about the convexity of the mapping $\left(\rho, \rho \varepsilon_{i}\right) \mapsto\left\{\rho s_{i}\right\}\left(\rho, \rho \varepsilon_{i}\right)$ for all $i=1, \ldots, N$, Jensen's inequality leads to

$$
\begin{aligned}
& \left\{\rho s_{i}\right\}\left(\bar{\rho}_{L}, \overline{\rho \varepsilon_{i}}\right) \leq 2 \lambda \int_{-1 / 2 \lambda}^{0}\left\{\rho s_{i}\right\}\left(\mathbf{w}\left(\xi, \mathbf{U}_{L}, \mathbf{U}_{R}\right)\right) d \xi \\
& \left\{\rho s_{i}\right\}\left(\bar{\rho}_{R}, \overline{\rho \varepsilon_{i}}\right) \leq 2 \lambda \int_{0}^{1 / 2 \lambda}\left\{\rho s_{i}\right\}\left(\mathbf{w}\left(\xi, \mathbf{U}_{L}, \mathbf{U}_{R}\right)\right) d \xi .
\end{aligned}
$$

Thus, entropy inequalities (105) are immediately obtained provided we mention (92). In addition, maximum principles (106) are also an immediate consequence of (107) thanks to (104). We now follow by establishing proposition 6, related to the step 1. Proof (Proposition 6)

First, invoking the convexity of the mapping $(\rho, \rho u) \mapsto \frac{(\rho u)^{2}}{2 \rho}$ and the definition (86)-(88)-(89), we obtain :

$$
\{\rho E\}\left(\mathbf{U}_{j}^{n+1-}\right):=\frac{\left((\rho u)_{j}^{n+1-}\right)^{2}}{2 \rho_{j}^{n+1-}}+\sum_{i=1}^{N}\left(\rho \varepsilon_{i}\right)_{j}^{n+1-} \leq \frac{1}{2}\left(\frac{\left(\overline{\rho u}_{L}\right)^{2}}{2 \bar{\rho}_{L}}+\frac{\left(\overline{\rho u}_{R}\right)^{2}}{2 \bar{\rho}_{R}}\right)+\sum_{i=1}^{N}\left(\rho \varepsilon_{i}\right)_{j}^{n+1-},
$$


so that Jensen's inequality and definitions (85) lead to

$$
\begin{gathered}
\{\rho E\}\left(\mathbf{U}_{j}^{n+1-}\right) \leq \sum_{i=1}^{N}\left(\rho \varepsilon_{i}\right)_{j}^{n+1-}+ \\
\frac{1}{2}\left(2 \lambda \int_{-1 / 2 \lambda}^{0}\left\{\frac{(\rho u)^{2}}{2 \rho}\right\}\left(\mathbf{w}\left(\xi, \mathbf{U}_{L}, \mathbf{U}_{R}\right)\right) d \xi+2 \lambda \int_{0}^{1 / 2 \lambda}\left\{\frac{(\rho u)^{2}}{2 \rho}\right\}\left(\mathbf{w}\left(\xi, \mathbf{U}_{L}, \mathbf{U}_{R}\right)\right) d \xi\right) .
\end{gathered}
$$

Arguing now about the averages (89)-(90), we easily obtain

$$
\begin{gathered}
\{\rho E\}\left(\mathbf{U}_{j}^{n+1-}\right) \leq \\
\frac{1}{2}\left(2 \lambda \int_{-1 / 2 \lambda}^{0}\{\rho E\}\left(\mathbf{w}\left(\xi, \mathbf{U}_{L}, \mathbf{U}_{R}\right)\right) d x+2 \lambda \int_{0}^{1 / 2 \lambda}\{\rho E\}\left(\mathbf{w}\left(\xi, \mathbf{U}_{L}, \mathbf{U}_{R}\right)\right) d x\right),
\end{gathered}
$$

that is to say

$$
\{\rho E\}\left(\mathbf{U}_{\mathbf{j}}^{\mathbf{n}+\mathbf{1},-}\right) \leq \frac{1}{2}\left({\overline{\{\rho E\}_{L}}}_{L}\left(\lambda ; \mathbf{U}_{j}^{n}, \mathbf{U}_{j+1}^{n}\right)+{\overline{\{\rho E\}_{R}}}_{R}\left(\lambda ; \mathbf{U}_{j-1}^{n}, \mathbf{U}_{j}^{n}\right)\right),
$$

or more precisely

$$
\{\rho E\}\left(\mathbf{U}_{j}^{n+1-}\right) \leq(\rho E)_{j}^{n}-\lambda\left(\{\rho \mathcal{H} u\}^{+}\left(\mathbf{U}_{j}^{n}, \mathbf{U}_{j+1}^{n}\right)-\{\rho \mathcal{H} u\}^{-}\left(\mathbf{U}_{j-1}^{n}, \mathbf{U}_{j}^{n}\right)\right),
$$

using the definitions (96) of $\{\rho \mathcal{H} u\}$. To conclude with the desired energy inequality (97), we juste have to recall the validity of $\{\rho \mathcal{H} u\}^{-} \leq\{\rho \mathcal{H} u\}^{+}$stated in the lemma 7 .

Concerning now the entropy inequalities (98), the convexity of the mapping $\left\{\rho s_{i}\right\}$ for all $i=1, \ldots, N$ and definitions (86)-(88)-(89) of $\rho_{j}^{n+1-}$ and $\left\{\left(\rho \varepsilon_{i}\right)_{j}^{n+1,-}\right\}_{i=1, \ldots, N}$, ensure that

$$
\left\{\rho s_{i}\right\}\left(\rho_{j}^{n+1-},\left(\rho \varepsilon_{i}\right)_{j}^{n+1,-}\right) \leq \frac{1}{2}\left(\left\{\rho s_{i}\right\}\left(\bar{\rho}_{L},{\overline{\rho \varepsilon_{i}}}_{L}\right)+\left\{\rho s_{i}\right\}\left(\bar{\rho}_{R}, \overline{\rho \varepsilon_{i}}\right)\right) .
$$

The required conclusion is then easily obtained by applying inequalities (105) of lemma 9 . Now, it remains to give the proof of theorem 8. Proof (Theorem 8)

Since by construction, the total energy $(\rho E)_{j}^{n+1}$ finds the conservative discrete formulation (101), we immediately infer from (97):

$$
\{\rho E\}\left(\mathbf{U}_{j}^{n+1-}\right)=\frac{\left((\rho u)_{j}^{n+1-}\right)^{2}}{2 \rho_{j}^{n+1-}}+\sum_{i=1}^{N}\left(\rho \varepsilon_{i}\right)_{j}^{n+1-} \leq(\rho E)_{j}^{n+1} .
$$

As a consequence, invoking the explicit formulae (102) for defining each of the internal energies $\left(\rho \varepsilon_{i}\right)_{j}^{n+1}$, the positivity of the viscosity coefficients easily ensures that :

$$
\left(\rho \varepsilon_{i}\right)_{j}^{n+1} \geq\left(\rho \varepsilon_{i}\right)_{j}^{n+1-} \text { for all } i \in\{1, \ldots, N\} .
$$

But, $\left(\rho \varepsilon_{i}\right)_{j}^{n+1-}$ stays by construction positive. Indeed, this quantity is nothing but the average (see indeed (89)-(90)) of the exact internal energy $\left(\rho \varepsilon_{i}\right)$ evaluated on approximate Riemann solutions under the CFL restriction (87). Such solutions stay within the admissible phase space and this gives the required conclusion.

Turning now establishing the entropy inequalities (103), we first recall that

$$
\frac{\partial \rho s_{i}}{\partial \rho \varepsilon_{i}}=-\frac{1}{T_{i}}<0
$$

to get for all $i=1, \ldots, N$ and thanks to (110) :

$$
\left(\rho s_{i}\right)_{j}^{n+1}=\left\{\rho s_{i}\right\}\left(\rho_{j}^{n+1},\left(\rho \varepsilon_{i}\right)_{j}^{n+1}\right) \leq\left\{\rho s_{i}\right\}\left(\rho_{j}^{n+1},\left(\rho \varepsilon_{i}\right)_{j}^{n+1,-}\right) .
$$

As an immediate consequence of (108) and for concluding, we now just quote lemma 9, namely (105) for the expected entropy inequalities and (106) for the maximum principles. 


\section{$6 \quad$ Numerical Experiments}

In this section, we perform numerical experiments for illustrating the correct design of the scheme (88)-(89)-(99)-(100)-(101) we have described in the previous section. Let us precise that we have used the Relaxation method described in[6] as a suitable Approximate Riemann Solver. We also refer the reader to[4] for additional numerical experiments.

To that purpose, we deal with polytropic ideal gases associated with $N$ constant adiabatic exponents $\gamma_{i}>1$ for all $i=1, \ldots, N$. As a consequence, observe in particular that the total energy now reads :

$$
\rho E=\frac{(\rho u)^{2}}{2 \rho}+\sum_{i=1}^{N} \frac{p_{i}}{\gamma_{i}-1} .
$$

The system (73) is solved with the following Riemann initial data :

$$
\mathbf{U}(x, 0)=\left\{\begin{array}{lll}
\mathbf{U}_{L} & \text { if } & x<0, \\
\mathbf{U}_{R} & \text { if } & x>0,
\end{array}\right.
$$

$\mathbf{U}_{L}$ and $\mathbf{U}_{R}$ to be prescribed in the phase space.

In this setting of $N$ constant viscosities for $N$ given polytropic gases, Chalons and Coquel[7] have shown existence and uniqueness (away from vacuum) of a Riemann solution for the limit system (36). More precisely, they have proved that the exit state $\mathbf{V}_{+}$connected in the future by a travelling wave solution issuing from a given state $\mathbf{V}_{-}$with properly prescribed velocity $\sigma$ can be entirely determined by simply solving a scalar nonlinear algebraic equation. Note that traveling wave solutions in[1] are numerically integrated after a convenient change of time-like variable (with compact range) so as to again allow for a proper resolution of the generalized jump conditions. The derivation proposed in [7] makes easier this task and so the construction of exact Riemann solutions. These results allow us to systematically compare our numerical solutions with exact ones.

We consider a 300-point mesh and plot the corresponding numerical solutions (see figures below) together with the exact ones and the ones given by a classical approach. Here "classical" means that we do not enforce for validity (22) (and then the generalized jump conditions (40)) from a numerical point of view. This is the very difference with the method proposed in the previous section.

\section{Experiment 1}

We set $N=3$ and choose $\gamma_{1}=\gamma_{2}=\gamma_{3}=1.4$ and $\mu_{2} / \mu_{1}=\mu_{3} / \mu_{1}=1$. The left and right states entering (111) write as follows :

$$
\begin{aligned}
& \left(\rho, u,\left\{p_{i}\right\}_{1 \leq i \leq 3}\right)_{L}=(2.0, \quad 1.0, \quad 1.0, \quad 1.2, \quad 1.4), \\
& \left(\rho, u,\left\{p_{i}\right\}_{1 \leq i \leq 3}\right)_{R}=\left(\begin{array}{lllll}
1.2838, & -1.3990, & 0.1850, & 0.1305, & 0.2550
\end{array}\right) \text {. }
\end{aligned}
$$

Since the adiabatic exponents are equal, it turns out that the system under study admits an equivalent full conservation form. More precisely, this system is nothing but the usual $3 \times 3$ Euler equations (with the pressure $\left.p:=\sum_{i=1}^{N} p_{i}\right)$ supplemented with $(N-1)$ additional independent transport equations (we refer the reader to[1] for such considerations). As a consequence, the classical approach should provide numerical solutions of $\rho, \rho u$ and $\rho E$ in full agreement with the exact ones. The difference may be located on the partial pressure laws only. Concerning our new approach, it turns out that each component fairly agrees with the exact solution, see figures 1,2 and 3 below. 

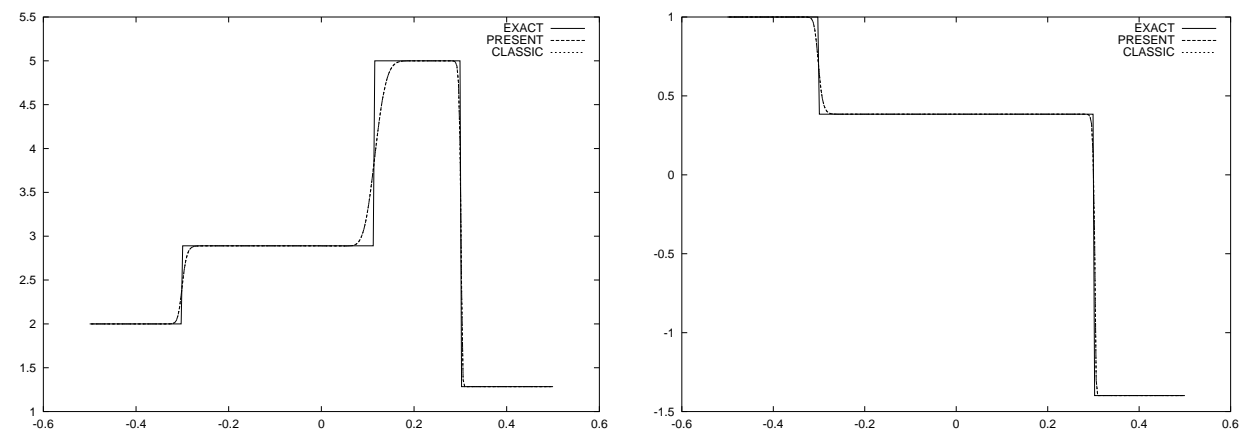

Figure 1: Density, Velocity

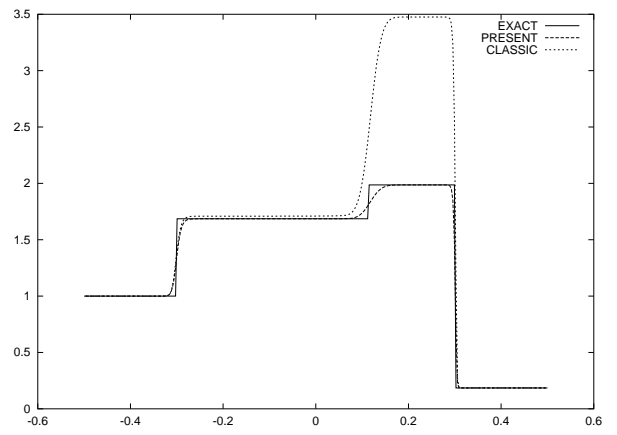

Figure 2: Pressure 1
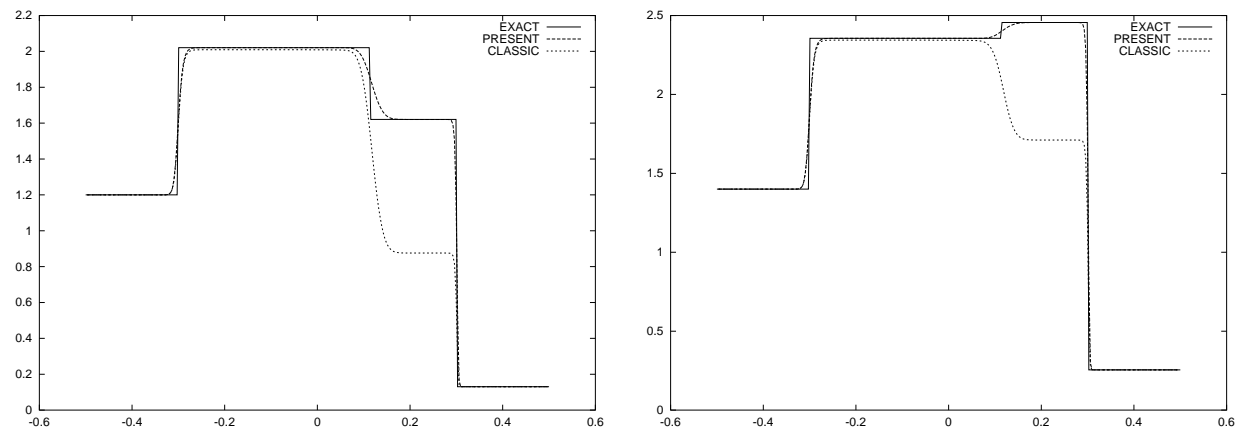

Figure 3: Pressure 2, Pressure 3 


\section{Experiment 2}

The adiabatic exponents are now different and the ratios of the viscosity laws no longer necessarily equal to 1 : we choose $\gamma_{1}=1.2, \gamma_{2}=1.4, \gamma_{3}=1.6$ and $\mu_{2} / \mu_{1}=1 ., \mu_{3} / \mu_{1}=100$. The left and right states entering (111) read as follows :

$$
\begin{aligned}
& \left(\rho, u,\left\{p_{i}\right\}_{1 \leq i \leq 3}\right)_{L}=(2.0, \quad 2.0, \quad 1.5, \quad 1.0, \quad 1.0), \\
& \left(\rho, u,\left\{p_{i}\right\}_{1 \leq i \leq 3}\right)_{R}=(3.3244, \quad-1.2999, \quad 1.4254, \quad 0.8667,0.0537) \text {. }
\end{aligned}
$$

Once more, we observe on figures 4, 5 and 6 that our new method provides us with good numerical results compared to the classical approach, in spite of the fact that this test case is difficult (the pressure jumps are quite large).
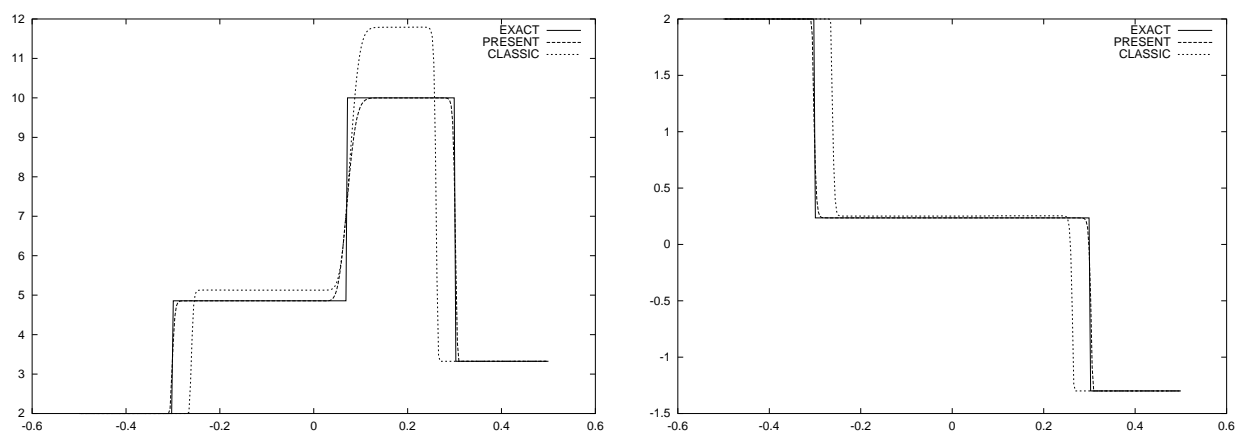

Figure 4: Density, Velocity

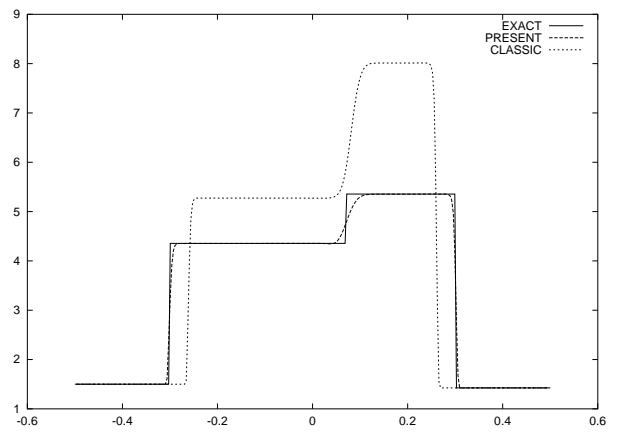

Figure 5: Pressure 1

Acknowledgments. The research of the first author has been supported by ONERA and a grant from DGA. The research of the second author has been supported in part by ONERA.

\section{References}

[1] Berthon C. and Coquel F., Nonlinear projection methods for multi-entropies Navier-Stokes systems, Innovative methods for numerical solutions of partial differential equations, Hafez M.M. et al. (eds), World Scientific, pp 278-304 (2002).

[2] Berthon C. and Coquel F. Travelling wave solutions of a convective diffusive system with first and second order terms in nonconservation form,Hyperbolic problems: theory, numerics, 

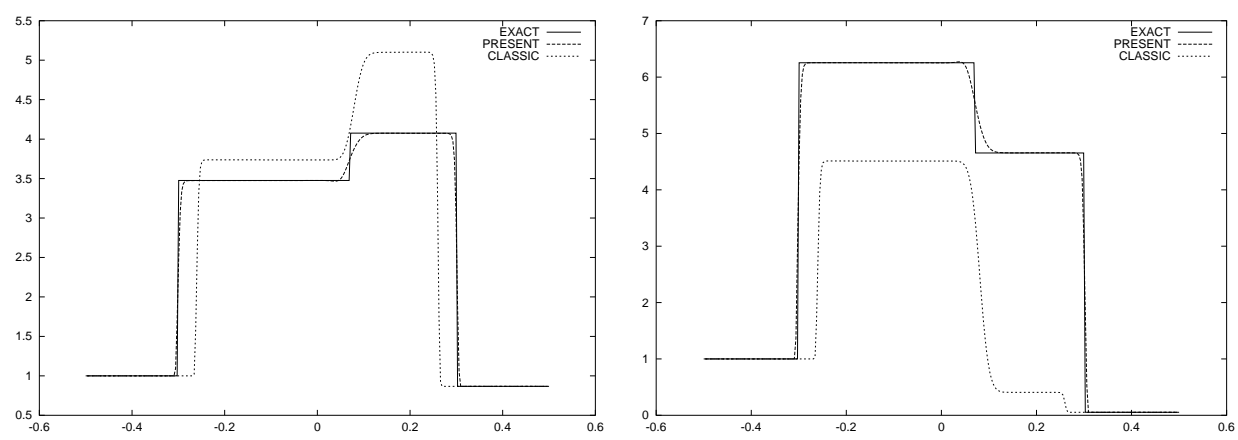

Figure 6: Pressure 2, Pressure 3

applications, Vol. I (Zrich, 1998), 47-54, Internat. Ser. Numer. Math., 129, Birkhauser, Basel, (1999).

[3] Berthon C., Coquel F. and LeFloch P.G., Entropy dissipation measure and kinetic relation associated with nonconservative hyperbolic systems, work in preparation.

[4] Chalons C., Bilans d'entropie discrets dans l'approximation numérique des chocs non classiques. Application aux équations de Navier-Stokes multi-pression $2 D$ et à quelques systèmes visco-capillaires, PhD Thesis, Ecole Polytechnique, (2002).

[5] Chalons C. and Coquel F., Numerical approximation of the Navier-Stokes equations with several independent specific entropies, Hyperbolic Problems : Theory, Numerics, Applications, Springer (2003).

[6] Chalons C. and Coquel F., Navier-Stokes equations with several independent pressure laws and explicit predictor-corrector schemes, to appear in Numerisch Math. (2005), available online since 05 September, 2005.

[7] Chalons C. and Coquel F., The Riemann problem for the multi-pressure Euler system, Journal of Hyperbolic Differential Equations, vol 2, No 3, pp 745-782 (2005)

[8] Chalons C. and LeFloch P.G., High-order entropy conservative schemes and kinetic relations for van der Waals fluids, J. Comput. Phys., vol 167, pp 1-23 (2001).

[9] Coquel F. and LeFloch P.G., Convergence of finite volumes schemes for scalar conservation laws in several space dimensions : a general theory, SIAM J. Numer. Anal., vol 30, pp 676-700 (1993).

[10] Colombeau J.F. and Leroux A.Y., Multiplications of distributions in elasticity and hydrodynamics, J. Math. Phys., vol 29, pp 315-319 (1988).

[11] Dal Maso G., LeFloch P.G. and Murat F., Definition and weak stability of a non conservative product, J. Math. Pures Appli., vol 74, pp 483-548 (1995).

[12] Godlewsky E. and Raviart P.A., Hyperbolic systems of conservation laws, Ellipse (1991).

[13] Godlewsky E. and Raviart P.A., Numerical approximation of hyperbolic systems of conservation laws, Springer (1995). 
[14] Harten A., Lax P.D. and Van Leer B., On upstream differencing and Godunov-type schemes for hyperbolic conservation laws, SIAM Rev., vol 25, pp 35-61 (1983).

[15] Hayes B.T. and LeFloch P.G., Nonclassical shocks and kinetic relations : Strictly hyperbolic systems, SIAM J. Math. Anal., vol 31, pp 941-991 (2000).

[16] Hou T.Y. and LeFloch P.G., Why nonconservative schemes converge to wrong solutions: error analysis, Math. of Comput. 62, pp 497-530 (1994).

[17] LeFloch P.G., Hyperbolic Systems of Conservation Laws: The theory of classical and nonclassical shock waves, E.T.H. Lecture Notes Series, Birkhäuser (2002).

[18] LeFloch P.G., Shock waves for nonlinear hyperbolic systems in nonconservative form, Institute for Math. and its Appl., Minneapolis, Preprint \# 593, (1989).

[19] Perthame B., Boltzmann type schemes for gaz dynamics and the entropy property, SIAM J. Numer. Anal., vol 27, pp 1405-1421 (1990).

[20] Raviart P.A. and Sainsaulieu L., A non conservative hyperbolic system modelling spray dynamics. I. Solution of the Riemann problem., Math. Models Methods Appl. Sci., vol 5, no. 3, pp 297-333 (1995).

[21] Roe P.L., Approximate Riemann solvers, parameter vectors and difference schemes, J. Comp. Phys., vol 43, pp 357-372 (1981). 